J. DIFFERENTIAL GEOMETRY

89 (2011) $87-110$

\title{
A NON-ARCHIMEDEAN ANALOGUE OF THE CALABI-YAU THEOREM FOR TOTALLY DEGENERATE ABELIAN VARIETIES
}

\author{
YIFENG LIU
}

\begin{abstract}
We show an example of a non-archimedean version of the existence part of the Calabi-Yau theorem in complex geometry. Precisely, we study totally degenerate abelian varieties and certain probability measures on their associated analytic spaces in the sense of Berkovich.
\end{abstract}

\section{Introduction}

The theorem of Calabi-Yau is one of the most important results in complex geometry and has many applications (e.g., Yau's famous paper [16]). In one version, it claims the following. Let $M$ be a compact complex manifold with an ample line bundle $L$. Then for any smooth positive measure $\mu$ on $M$ with $\int_{M} \mu=\int_{M} c_{1}(L)^{\wedge \text { dim } M}$, there is a positive metric \|\| on $L$, unique up to a constant multiple, such that $c_{1}(L,\|\|)^{\wedge \operatorname{dim} M}=\mu$.

One would like to ask a similar question for a non-archimedean field, for example, if we replace $\mathbb{C}$ by $\mathbb{C}_{p}$ and complex manifolds by nonarchimedean analytic spaces, or Berkovich spaces in $[\mathbf{1}]$. Hence let $X$ be the analytic space associated to a projective variety over $\mathbb{C}_{p}$ equipped with (the analytification of) an ample line bundle $L$. Given any integrable metric \|\| (cf. [21]) on $L$, although we do not have a nice analogue of $(1,1)$-form for $c_{1}(L,\|\|)$ in the non-archimedean situation so far, we can still talk about its top wedge product, i.e., $c_{1}(L,\|\|)^{\wedge \operatorname{dim} M}$ which is defined by Chambert-Loir in [5]. The top wedge product is a measure on the underlying compact (metrizable) topological space of $X$. If \|\| is semipositive in the sense of $[\mathbf{2 0 , 2 1}]$, then $c_{1}(L,\|\|)^{\wedge \operatorname{dim} M}$ is a positive measure in the following sense: $\int_{X} f c_{1}(L,\|\|)^{\wedge \operatorname{dim} M} \geq 0$ for any non-negative continuous real function $f$ on $X$. Then analogously to the complex case, given a positive measure $\mu$ on $X$ with $\int_{X} \mu=\operatorname{deg}_{L}(X)$, we can ask the following two questions:

Received 11/30/2010. 
(E): Does there exist a semipositive metric \|\| on $L$ such that

$$
c_{1}(L,\|\|)^{\wedge \operatorname{dim} M}=\mu ?
$$

(U): If it does, is it unique up to a constant multiple?

The question (U) has been answered positively by Xinyi Yuan and Shou-Wu Zhang in [18]. Moreover, they have already used this uniqueness result to prove several exciting theorems in algebraic dynamic systems. The answer to the question (E) is known to be positive for curves and measures compactly supported on type II or III points, or the limits of such measures, which is implied by the theory in [19]. In any dimension, there is a necessary condition for the equality in (E) to hold, due to a result of Chambert-Loir and Thuillier (cf. [6, corollaire 4.2]), that $\mu$ cannot be supported on a proper algebraic subset of $X$, which is well-known in the archimedean case. But even under this restriction, the answer to (E) is still negative in general, due to several reasons. One conceptual reason is that the notions of being positive or smooth in the metric side and the measure side are not quite compatible as in the complex case. We are still not clear about the nature of this question, especially for its correct formulation. Nevertheless, we would like to give one example where the answer to (E) is positive (of course, under certain restrictions).

Let us consider a totally degenerate abelian variety $A$, say over $\mathbb{C}_{p}$, i.e., the special fibre of its Néron model is a torus, which is also equivalent to saying that the associated analytic space $A^{\text {an }}$ is isomorphic to a quotient $\left(\mathbb{G}_{m}^{d}\right)^{\text {an }} / M$ for a complete lattice $M \subset \mathbb{G}_{m}^{d}\left(\mathbb{C}_{p}\right)$ (cf. Section $1)$. We have an evaluation map $\tau_{A}: A^{\text {an }}=\left(\mathbb{G}_{m}^{d}\right)^{\text {an }} / M \rightarrow \mathbb{R}^{d} / \Lambda$ with a complete lattice $\Lambda \subset \mathbb{R}^{d}$, which is continuous and surjective. This map has a continuous section $i_{A}: \mathbb{R}^{d} / \Lambda \hookrightarrow A^{\text {an }}$. In fact, $i_{A}$ identifies $\mathbb{R}^{d} / \Lambda$ as a strong deformation retract of $A^{\text {an }}$ for which $i_{A} \circ \tau_{A}=\Phi(\cdot, 1)$ for a strong retraction map $\Phi: A^{\text {an }} \times[0,1] \rightarrow A^{\text {an }}$. Then we prove the following theorem, which is a certain non-archimedean analogue of the Calabi-Yau theorem.

Theorem (4.2). Let $A$ be a d-dimensional totally degenerate abelian variety over $k$ (e.g. $\mathbb{C}_{p}$ ) and $L \in \operatorname{Pic}(A) \otimes_{\mathbb{Z}} \mathbb{Q}$ an ample class on $A$. For any measure $\mu=f \mathrm{~d} \mathbf{x}$ of $\mathbb{R}^{d} / \Lambda$ with $f$ a strictly positive smooth function, $\mathrm{d} \mathbf{x}$ the Lebesgue measure on $\mathbb{R}^{d} / \Lambda$, and $\int_{\mathbb{R}^{d} / \Lambda} \mu=\operatorname{deg}_{L}(A)$, there is a semipositive metric \|\| on $L$, unique up to a constant multiple, such that $c_{1}(\bar{L})^{d}=\left(i_{A}\right)_{*} \mu$ where $\bar{L}=(L,\|\|)$.

The main ingredient of the proof is a limit formula for the measure associated to certain integrable metrics, which is Theorem 3.3. According to this formula, the existence part ends up with a (real) Monge-Ampère equation on a real torus, quite similar to the complex case, although we are doing non-archimedean geometry. In fact, let $\mathbb{R}^{d} / \Lambda$ be the real 
torus as above with usual coordinate $x_{1}, \ldots, x_{d}$ and Lebesgue measure $\mathrm{d} \mathbf{x}=\mathrm{d} x_{1} \cdots \mathrm{d} x_{d}$. Let $\left(g_{i j}\right)_{i, j=1, \ldots, d}$ be a positive definite (real symmetric) matrix. Then for any smooth real function $f$ on $\mathbb{R}^{d} / \Lambda$ such that $\int_{\mathbb{R}^{d} / \Lambda} \mathrm{e}^{f} \mathrm{~d} \mathbf{x}=\operatorname{covol}(\Lambda)$, we will show that there exists a unique smooth (real) function $\phi$ on $\mathbb{R}^{d} / \Lambda$ such that:

- the matrix $\left(g_{i j}+\frac{\partial^{2} \phi}{\partial x_{i} \partial x_{j}}\right)$ is positive definite;

- $\int_{\mathbb{R}^{d} / \Lambda} \phi \mathrm{d} \mathbf{x}=0$;

- it satisfies the following real Monge-Ampère equation:

$$
\operatorname{det}\left(g_{i j}+\frac{\partial^{2} \phi}{\partial x_{i} \partial x_{j}}\right)=\operatorname{det}\left(g_{i j}\right) \cdot \mathrm{e}^{f} .
$$

The above type of real Monge-Ampère equation has been solved by Cheng-Yau in $[\mathbf{7}, \S 2]$ without invoking the complex one. There is a second solution (see $[\mathbf{7}, \S 3]$ ) which applies to more general types of real Monge-Ampère equations on compact Kähler affine flat manifolds, using a tube domain construction and reducing to the complex case which has already been solved by Yau $[\mathbf{1 7}]$ when attacking the (classical) Calabi conjecture. Hence the same PDE problem applies to this nonarchimedean Calabi-Yau theorem as well. We will resolve the equation along the second line for our special case here, not only in order of this PDE problem, but also to suggest relations between archimedean (complex) and non-archimedean pictures.

At last, we would like to make some remarks about notations. We use | | for non-archimedean norms; || || for metrics on line bundles. But due to the conventions, we will also use || for the usual absolute value of real numbers and total measures; \|\| for the Euclidean norm of $\mathbb{R}^{d}$ when $d>1$.

For simplicity, we will assume that $k$ is the completion of the algebraic closure of a $p$-adic local field, i.e., isomorphic to $\mathbb{C}_{p}$ or $\overline{\mathbb{F}_{p}((t))}$, the completion of the algebraic closure of $\mathbb{F}_{p}((t))$. Such restriction on $k$ is unnecessary. In fact, all results and argument remain valid for any algebraically closed non-archimedean Banach field whose valuation is non-trivial: for example, the completion of the algebraic closure of $\mathbb{C}((t))$. Then one only needs to use the generalized definition of Chambert-Loir's measure given by Gubler in $[\mathbf{1 1}, \S 3]$.

Acknowledgements. This paper is motivated by works of Xinyi Yuan and Shou-Wu Zhang [18] on the uniqueness part of the non-archimedean Calabi-Yau theorem, and Gubler [11] on the tropical geometry of totally degenerate abelian varieties. The author would like to thank Xander Faber, Shing-Tung Yau, Xinyi Yuan, and Shou-Wu Zhang for stimulating discussions. This work was presented in the Number Theory Seminar at Harvard University and AMS Sectional Meeting \#1065, Special Session on Applications of Non-Archimedean Geometry. The author 
thanks the organizers of both. Finally, the author would like to thank referees for their helpful comments.

\section{Mumford's Construction}

In this section, we briefly recall Mumford's construction of (formal) models of totally degenerate abelian varieties in $[\mathbf{1 5}, \S 6]$; see also $[\mathbf{1 1}$, $\S 4, \S 6]$.

1.1. Valuation map. Let $k$ be the completion of the algebraic closure of a $p$-adic local field. Let || be the norm on $k$ and its extended valuation fields, $k^{\circ}$ the subring of $k$ consisting of elements $x \in k$ with $|x| \leq 1, k^{\circ \circ}$ the maximal ideal of $k^{\circ}$ consisting of elements $x \in k$ with $|x|<1$, and $\widetilde{k}=k^{\circ} / k^{\circ \circ}$ the residue field which is algebraically closed. We fix a $\operatorname{logarithm} \log$ such that $\log |x| \in \mathbb{Q}$ for all $x \in k^{\times}$.

Fix a split torus $T=\mathbb{G}_{m, k}^{d}$ of rank $d \geq 1$ over $k$. We have the following valuation map:

$$
\tau: T^{\text {an }}=\left(\mathbb{G}_{m, k}^{d}\right)^{\text {an }} \longrightarrow \mathbb{R}^{d}, \quad t \mapsto\left(-\log \left|T_{1}(t)\right|, \ldots,-\log \left|T_{d}(t)\right|\right)
$$

where $\left(\mathbb{G}_{m, k}^{d}\right)^{\text {an }}$ is the associated $k$-analytic space (cf. [1, $\left.\left.\S 3.4\right]\right)$ and $T_{i}$ are coordinate functions. It is surjective and continuous with respect to the underlying topology of the analytic space and the usual topology of $\mathbb{R}^{d}$. Moreover, $\tau$ has a continuous section

$$
i: \mathbb{R}^{d} \longrightarrow T^{\mathrm{an}}, \quad \mathbf{x} \mapsto \xi_{\mathbf{x}}
$$

where $\xi_{\mathrm{x}}$ is (the unique point of) the Shilov boundary of the affinoid domain $\tau^{-1}(\mathbf{x})$ (cf. [11, corollary 4.5]). Precisely, the $k$-affinoid algebra associated to $\tau^{-1}(\mathbf{x})$ is

$$
k\left\langle\tau^{-1}(\mathbf{x})\right\rangle=\left\{\sum_{\mathbf{m} \in \mathbb{Z}^{d}} a_{\mathbf{m}} u_{1}^{m_{1}} \cdots u_{d}^{m_{d}}\left|\lim _{|\mathbf{m}| \rightarrow \infty}-\log \right| a_{\mathbf{m}} \mid+\mathbf{m} \cdot \mathbf{x}=\infty\right\}
$$

and the point $\xi_{\mathbf{x}}$ corresponds to the norm

$$
\left|\sum_{\mathbf{m} \in \mathbb{Z}^{d}} a_{\mathbf{m}} \mathbf{u}^{\mathbf{m}}\right|=\max _{\mathbf{m} \in \mathbb{Z}^{d}}\left|a_{\mathbf{m}}\right| \mathrm{e}^{-\mathbf{m} \cdot \mathbf{x}} .
$$

Now consider a totally degenerate abelian variety $A$ over $k$, i.e., the special fibre of its Néron model is a torus. Then $A^{\text {an }} \cong T^{\text {an }} / M$ for a complete lattice $M \subset T(k)$. By a complete lattice, we mean that $M$ is a (discrete free) subgroup of $T(k)$ and maps bijectively to a rational complete lattice $\Lambda \subset \mathbb{R}^{d}$ under $\tau$. Here, we say a complete lattice is rational if it has a basis whose coordinates are in $\mathbb{Q}$. Hence we have the induced map $\tau_{A}: A^{\text {an }} \rightarrow \mathbb{R}^{d} / \Lambda$ and $i_{A}: \mathbb{R}^{d} / \Lambda \hookrightarrow A^{\text {an }}$. In fact, $i_{A}$ identifies $\mathbb{R}^{d} / \Lambda$ with a strong deformation retract or a skeleton of $A^{\text {an }}$ as in $[\mathbf{1}, \S 6.5]$. 
1.2. Rational polytopes. A compact subset $\Delta$ of $\mathbb{R}^{d}$ is called a polytope if it is an intersection of finitely many half-spaces $\left\{\mathbf{x} \in \mathbb{R}^{d} \mid \mathbf{m}_{i} \cdot \mathbf{x} \geq\right.$ $\left.c_{i}\right\}$. We say $\Delta$ is rational if we can choose all $\mathbf{m}_{i} \in \mathbb{Z}^{d}$ and $c_{i} \in \mathbb{Q}$. The $\operatorname{dimension} \operatorname{dim}(\Delta)$ of $\Delta$ is its usual topological dimension and we denote by $\operatorname{int}(\Delta)$ the topological interior of $\Delta$ in $\mathbb{R}^{d}$. A closed face of $\Delta$ is either $\Delta$ itself or $B \cap \Delta$ where $B$ is the boundary of a half-space containing $\Delta$. It is obvious that a closed face of a (rational) polytope is again a (rational) polytope. An open face is a closed face with all its properly contained closed faces removed.

A (rational) polytopal complex $\mathcal{C}$ in $\mathbb{R}^{d}$ is a locally finite set of (rational) polytopes such that (1) if $\Delta \in \mathcal{C}$, then all its closed faces are in $\mathcal{C}$ and (2) if $\Delta, \Delta^{\prime} \in \mathcal{C}$, then $\Delta \cap \Delta^{\prime}$ is either empty or a closed face of both $\Delta$ and $\Delta^{\prime}$. The polytopes of dimension 0 are called vertices. We say $\mathcal{C}$ is a (rational) polytopal decomposition of $S \subset \mathbb{R}^{d}$ if $S$ is the union of all polytopes in $\mathcal{C}$. In particular, if $S=\mathbb{R}^{d}$, we say $\mathcal{C}$ is a (rational) polytopal decomposition of $\mathbb{R}^{d}$.

For a (rational) complete lattice $\Lambda \subset \mathbb{R}^{d}$, we say $\mathcal{C}$ is $\Lambda$-periodic if $\Delta \in \mathcal{C}$ implies $\Delta+\lambda \in \mathcal{C}$ for all $\lambda \in \Lambda$. A (rational) polytopal decomposition $\mathcal{C}_{\Lambda}$ of $\mathbb{R}^{d} / \Lambda$ for a (rational) complete lattice $\Lambda$ is a $\Lambda$ periodic (rational) polytopal decomposition $\mathcal{C}$ of $\mathbb{R}^{d}$ such that $\Delta$ maps bijectively to its image under the projection $\mathbb{R}^{d} \rightarrow \mathbb{R}^{d} / \Lambda$ for all $\Delta \in \mathcal{C}$. A polytope, a closed face, or an open face of $\mathcal{C}_{\Lambda}$ is a $\Lambda$-translation equivalence class of the corresponding object of $\mathcal{C}$.

A continuous real function $f$ on $\mathbb{R}^{d}$ is called (rational) polytopal if there is a (rational) polytopal decomposition $\mathcal{C}$ of $\mathbb{R}^{d}$ such that $f$ restricted to all $\Delta \in \mathcal{C}$ is affine (and takes rational values on all vertices). We denote by $\mathscr{C}_{\text {poly }}\left(\mathbb{R}^{d}\right)\left(\mathscr{C}_{\text {rpoly }}\left(\mathbb{R}^{d}\right)\right)$ the space of (rational) polytopal continuous functions on $\mathbb{R}^{d}$. We have the following simple lemma. Moreover, $f$ is called strongly polytopal convex with respect to $\mathcal{C}$ if it is convex and the maximal connected subsets on which $f$ is affine are $\Delta \in \mathcal{C}$ with $\operatorname{dim}(\Delta)=d$.

Lemma 1.1. Let $\Lambda$ be a (rational) complete lattice of $\mathbb{R}^{d}$ and $f$ in $\mathscr{C}_{\text {poly }}\left(\mathbb{R}^{d}\right)\left(\mathscr{C}_{\text {rpoly }}\left(\mathbb{R}^{d}\right)\right)$ satisfying

1) there exist affine functions $z_{\boldsymbol{\lambda}}$ for all $\boldsymbol{\lambda} \in \Lambda$ satisfying $f(\mathbf{x}+\boldsymbol{\lambda})=$ $f(\mathbf{x})+z_{\boldsymbol{\lambda}}(\mathbf{x})$ for all $\boldsymbol{\lambda}$ and $\mathbf{x} \in \mathbb{R}^{d}$;

2) if $\Delta$ is any maximal connected subset on which $f$ is affine, then $\Delta$ is a bounded convex subset of $\mathbb{R}^{d}$.

Then such $\Delta$ is a (rational) polytope and if $\mathcal{C}$ is the polytopal complex generated by all such $\Delta$ and their closed faces, then $\mathcal{C}$ is a $\Lambda$-periodic (rational) polytopal decomposition of $\mathbb{R}^{d}$.

Proof. Since $\Delta$ is closed, it is compact by (2). Since $f$ is (rational) polytopal, there is a finite (rational) polytopal decomposition of $\Delta$; hence $\Delta$ itself is a (rational) polytope since it is convex. Let $\mathcal{C}$ 
be the (rational) polytopal complex generated by all such $\Delta$ and their closed faces, which is obviously a polytopal decomposition of $\mathbb{R}^{d}$. The $\Lambda$-periodicity is implied by (1).

q.e.d.

1.3. Formal models. Let $X$ be a projective scheme over $k$; a $k^{\circ}$-model $\mathscr{X}$ of $X$ is a scheme projective and flat over Spec $k^{\circ}$ whose generic fibre $\mathscr{X}_{\eta} \cong X$. A formal $k^{\circ}$-model $\mathcal{X}$ of $X$ is an admissible formal scheme over $\operatorname{Spf} k^{\circ}$ (cf. $\left.[\mathbf{1 1}, 2.6]\right)$ whose generic fibre $\mathcal{X}_{\eta} \cong X^{\text {an }}$ in the sense of analytic spaces (cf. $[\mathbf{2}, \S 1])$. We denote by $\widetilde{\mathscr{X}}$ (resp. $\widetilde{\mathcal{X}}$ ) the special fibre of $\mathscr{X}$ (resp. $\mathcal{X}$ ) which is a proper scheme over Spec $\widetilde{k}$. The following result is due to Mumford in the case that $A$ is the base change of an abelian variety over a $p$-adic local field (cf. [15, corollary 6.6]) and extended by Gubler in the general case (cf. [11, proposition 6.3]).

Proposition 1.2. Given a rational polytopal decomposition $\mathcal{C}_{\Lambda}$ of $\mathbb{R}^{d} / \Lambda$, we may associate a formal $k^{\circ}$-model $\mathcal{A}$ of $A$ whose special fibre $\widetilde{\mathcal{A}}$ is reduced and the irreducible components $Y$ of $\widetilde{\mathcal{A}}$ are toric varieties and one-to-one correspond to the vertices $\mathbf{v}$ of $\mathcal{C}_{\Lambda}$ by $\mathbf{v}=\tau_{A}\left(\xi_{Y}\right)$, where $\xi_{Y} \in A^{\text {an }}$ is the point corresponding to $Y$. The formal scheme $\mathcal{A}$ has a covering by formal open affine sets $\mathcal{U}_{\Delta}$ for $\Delta \in \mathcal{C}_{\Lambda}$. Moreover, if $A$ is the base change of an abelian variety over a p-adic local field, then $\mathcal{A}$ can be constructed as a $k^{\circ}$-model.

\section{Toric Metrized Line Bundles}

In this section, we briefly recall the theory of metrized line bundles and their associated measure for general varieties. We introduce line bundles and toric metrized line bundles on $A$. Then we prove the main result, identifying certain toric integrable metrics.

2.1. Metrized line bundles and measure. The general theory of metrized line bundles is developed in [20], [21]; see also [5] and [10]. Let $X$ be a projective scheme over $k$, and $L$ a line bundle over $X$ (or more generally, a class in $\left.\operatorname{Pic}(X) \otimes_{\mathbb{Z}} \mathbb{Q}\right)$. A metric \|\| on $L$ assigns to every open subset $U$ of $X^{\text {an }}$ and a section $s \in \Gamma\left(U, L^{\text {an }}\right)$, a continuous function $\|s\|_{U}: U \rightarrow \mathbb{R}_{\geq 0}$ such that

1) $\|f s\|_{U}=|f| \cdot\|s\|_{U}$ for all $f \in \Gamma\left(U, \mathcal{O}_{U}\right)$;

2) $\|s\|_{U}(x)=0$ if and only if $s(x)=0$ for $x \in U$;

3) for any other $s^{\prime} \in \Gamma\left(U^{\prime}, L^{\text {an }}\right)$ such that $\left.s^{\prime}\right|_{U \cap U^{\prime}}=\left.s\right|_{U \cap U^{\prime}}$, we have $\left.\left\|s^{\prime}\right\|_{U^{\prime}}\right|_{U \cap U^{\prime}}=\left.\|s\|_{U}\right|_{U \cap U^{\prime}}$.

We say a metric is algebraic if it is defined by a model $(\mathscr{X}, \mathscr{L})$ where $\mathscr{X}$ is a $k^{\circ}$-model of $X$ and $\mathscr{L}$ is a line bundle on $\mathscr{X}$ such that $\mathscr{L}_{\eta} \cong L^{e}$ for some integer $e \geq 1$. A metric is formal if we replace the $k^{\circ}$-model $\mathscr{X}$ by a formal $k^{\circ}$-model $\mathcal{X}$, and $\mathscr{L}$ by a formal line bundle $\mathcal{L}$ on $\mathcal{X}$ such that $\mathcal{L}_{\eta} \cong\left(L^{e}\right)^{\text {an }}$. In fact, all formal metrics are algebraic. An algebraic (resp. formal) metric is called semipositive if $\mathscr{L}$ (resp. $\mathcal{L}$ ) has 
non-negative degree on every closed curve on the special fibre $\widetilde{\mathscr{X}}$ (resp. $\widetilde{\mathcal{X}}$ ) over $k^{\circ}$. In general, a metric on $L$ is called semipositive if it is the uniform limit of algebraic semipositive metrics. A metrized line bundle is called integrable if it is isomorphic to a quotient of two semipositive metrized line bundles.

Next we recall the construction of measures by Chambert-Loir in $[\mathbf{5}$, $\S 2]$. For simplicity, we only recall the algebraic case (which we only need for calculation later), and for the general case, one needs to pass to the limit, for which we refer to $[\mathbf{5}, \S 2]$ for details. Let $X$ be as above of dimension $d \geq 1$, and consider $d$ classes $L_{i} \in \operatorname{Pic}(X) \otimes_{\mathbb{Z}} \mathbb{Q}(i=1, \ldots, d)$ of line bundles. We endow $L_{i}$ with an algebraic measure \|\|$_{i}$ induced by $\left(\mathscr{X}, \mathscr{L}_{i}\right)$ with $\left(\mathscr{L}_{i}\right)_{\eta} \cong L_{i}^{e_{i}}$ on a common model $\mathscr{X}$ which is assumed to be normal. Let $Y_{j}$ be the reduced irreducible components of $\widetilde{\mathscr{X}}$ and $\xi_{j}$ the unique point in the inverse image of the generic point of $Y_{j}$ under the reduction map $\pi: X^{\text {an }} \rightarrow \widetilde{\mathscr{X}}$. Then we define

$$
c_{1}\left(\overline{L_{1}}\right) \wedge \cdots \wedge c_{1}\left(\overline{L_{d}}\right)=\frac{1}{e_{1} \cdots e_{d}} \sum_{j} m_{j}\left(c_{1}\left(\mathscr{L}_{1}\right) \cdots c_{1}\left(\mathscr{L}_{d}\right) \mid Y_{j}\right) \delta_{\xi_{j}}
$$

where $\overline{L_{i}}=\left(L_{i},\|\|_{i}\right), m_{j}$ is the multiplicity of $Y_{j}$ in $\widetilde{\mathscr{X}}$ and $\delta_{\xi_{j}}$ is the normalized Dirac measure supported at $\xi_{j}$. In general, the measure $c_{1}\left(\overline{L_{1}}\right) \wedge \cdots \wedge c_{1}\left(\overline{L_{d}}\right)$ is symmetric and $\mathbb{Q}$-multilinear, and we have

$$
\int_{X^{\mathrm{an}}} c_{1}\left(\overline{L_{1}}\right) \wedge \cdots \wedge c_{1}\left(\overline{L_{d}}\right)=c_{1}\left(L_{1}\right) \cdots c_{1}\left(L_{d}\right) \mid X .
$$

2.2. Line bundles on $A$. The theory of line bundles on totally degenerate abelian varieties is very similar to that over the complex field. We refer to $[\mathbf{3}, \S 2]$ and $[\mathbf{8}$, chapter 6$]$ for more details.

Let $A$ be a totally degenerate abelian variety as above and $\check{M}=$ $\operatorname{Hom}_{k}\left(T, \mathbb{G}_{m, k}\right)$ the character group of $T$. Let $\check{T}$ be the split torus with character group $M$, i.e., $\check{T}=\operatorname{Hom}_{k}\left(M, \mathbb{G}_{m, k}\right)$. Then $\check{A}^{\text {an }}$ is canonically isomorphic to $\check{T}^{\text {an }} / \check{M}$ where $\check{A}$ is the dual abelian variety of $A$. Let $L$ be a line bundle on $A$; the pull-back of $L^{\text {an }}$ to $T^{\text {an }}$ is trivial and is identified with $T^{\mathrm{an}} \times \mathbb{G}_{a, k}^{\mathrm{an}}$. Hence $L^{\text {an }}$ is identified with a quotient $\left(T^{\mathrm{an}} \times\right.$ $\left.\mathbb{G}_{a, k}^{\mathrm{an}}\right) / M$ where the action of $M$ on $T^{\text {an }}$ is given by an element $\mu \mapsto Z_{\mu}$ of $\mathrm{H}^{1}\left(M, \mathcal{O}(T)^{\times}\right)$. The function $Z_{\mu}$ has the form $Z_{\mu}=d_{\mu} \sigma_{\mu}$ where $d_{\mu} \in$ $k^{\times}, \mu \mapsto \sigma_{\mu}$ is a group homomorphism $\sigma: M \rightarrow \check{M}$ and $d_{\mu \nu} d_{\mu}^{-1} d_{\nu}^{-1}=$ $\sigma_{\nu}(\mu)$. By the isomorphism $\tau: M \rightarrow \Lambda$, we get a unique symmetric bilinear form $b$ on $\mathbb{R}^{d}$ such that $b(\tau(\mu), \tau(\nu))=-\log \left|\sigma_{\nu}(\mu)\right|$. Then $b$ is positive definite if and only if $L$ is ample. Since $\sigma_{\mu}$ is a character, $\left|Z_{\mu}\right|$ factors through $\tau$ and hence uniquely determines a function $z_{\lambda}$ on $\mathbb{R}^{d}$, such that $z_{\boldsymbol{\lambda}}(\tau(t))=-\log \left|Z_{\mu}(t)\right|$ for all $\mu \in M$ and $t \in T$, where 
$\boldsymbol{\lambda}=\tau(\mu)$. The function $z_{\boldsymbol{\lambda}}$ is affine with

$$
z_{\boldsymbol{\lambda}}(\mathbf{x})=z_{\boldsymbol{\lambda}}(\mathbf{0})+b(\mathbf{x}, \boldsymbol{\lambda}), \quad \boldsymbol{\lambda} \in \Lambda, \mathbf{x} \in \mathbb{R}^{d} .
$$

By linearity, we can also assign $b$ and $z_{\boldsymbol{\lambda}}$ to any class $L \in \operatorname{Pic}(A) \otimes_{\mathbb{Z}} \mathbb{Q}$.

Before stating the next lemma, we introduce some notations. We fix a $\mathbb{Z}$-basis $\left(\boldsymbol{\lambda}_{1}, \ldots, \boldsymbol{\lambda}_{d}\right)$ of $\Lambda$ once for all and let $\mathfrak{F}=\left\{\mathbf{x}=x_{1} \boldsymbol{\lambda}_{1}+\cdots+\right.$ $\left.x_{d} \boldsymbol{\lambda}_{d} \mid 0 \leq x_{i}<1\right\}$ be a fundamental domain of $\Lambda$. The volume of the closure $\overline{\mathfrak{F}}$ under the usual Lebesgue measure $\mathrm{d} \mathbf{x}$ of $\mathbb{R}^{d}$ only depends on $\Lambda$ and will be denoted by $\operatorname{covol}(\Lambda)$. We define $R_{\mathfrak{F}}=\max _{\mathbf{x}, \mathbf{x}^{\prime} \in \overline{\mathfrak{F}}}\left\|\mathbf{x}-\mathbf{x}^{\prime}\right\|$ and $r_{\mathfrak{F}}$ to be the maximal radius of balls contained in $\overline{\mathfrak{F}}$. We denote by $\mathbb{S}^{d-1} \subset \mathbb{R}^{d}$ the standard unit ball, $\mathbf{S O}_{d}$ the special orthogonal group of $\mathbb{R}^{d}$, and $\mathfrak{S}_{d}$ the group of $d$-permutations.

Let $\left(\mathbf{e}_{1}, \ldots, \mathbf{e}_{d}\right)$ be the standard basis of the Euclidean space $\mathbb{R}^{d}$, $\mathscr{C}^{l}\left(\mathbb{R}^{d}\right)\left(l \geq 0\right.$ and $\left.\mathscr{C}=\mathscr{C}^{0}\right)$ the space of real functions whose $l$-th partial derivatives exist and are continuous, $\mathscr{C}^{\infty}\left(\mathbb{R}^{d}\right)$ the space of real smooth functions, and $\mathscr{C}^{k, \alpha}\left(\mathbb{R}^{d}\right)(k \geq 0, \alpha \in[0,1))$ the spaces of real functions whose $k$-th partial derivatives exist and are Hölder continuous with exponential $\alpha$. We denote by $\mathscr{C}_{>0}^{l}\left(\mathbb{R}^{d}\right)$ the subspace of functions non-negative everywhere and $\mathscr{C}_{>0}^{l}\left(\mathbb{R}^{d}\right)$ that of functions positive everywhere; similarly, we have $\mathscr{C}_{>0}^{\infty}\left(\mathbb{R}^{d}\right), \mathscr{C}_{>0}^{\infty}\left(\mathbb{R}^{d}\right), \mathscr{C}_{>0}^{k, \alpha}\left(\mathbb{R}^{d}\right)$, and $\mathscr{C}_{>0}^{k, \alpha}\left(\mathbb{R}^{d}\right)$. For a certain function $f$, we write $f_{i}=\nabla_{\mathbf{e}_{i}} f, f_{i j}=\nabla_{\mathbf{e}_{i}} \nabla_{\mathbf{e}_{j}} f$, and $f_{i j k}=\nabla_{\mathbf{e}_{i}} \nabla_{\mathbf{e}_{j}} \nabla_{\mathbf{e}_{k}} f$ if the corresponding directional derivatives exist.

Let $q(\mathbf{x})=\frac{1}{2} b(\mathbf{x}, \mathbf{x})$ be the associated quadratic form and

$$
H_{q}=d ! \operatorname{det}\left(q_{i j}\right)_{i, j=1, \ldots, d}
$$

which is a constant associated to $q$. We have the following lemma.

Lemma 2.1. If $L \in \operatorname{Pic}(A) \otimes_{\mathbb{Z}} \mathbb{Q}$ is an ample class, then $\operatorname{deg}_{L}(A)=$ $\operatorname{covol}(\Lambda) H_{q}$.

Proof. Consider the morphism $\phi_{L}: A \rightarrow \check{A}$ associated to $L$. Its lifting $T \rightarrow \check{T}$ restricts to $\mu: M \rightarrow \check{M}$ on $M$. It is easy to see that $\mu$ is injective since $L$ is ample and

$$
\begin{aligned}
\operatorname{deg}(\mu)=[\check{M}: \mu(M)] & =\operatorname{covol}(\Lambda)^{-1} \operatorname{det}\left(b\left(\boldsymbol{\lambda}_{i}, \boldsymbol{\lambda}_{j}\right)\right)_{i, j=1, \ldots, d} \\
& =\operatorname{covol}(\Lambda) \operatorname{det}\left(q_{i j}\right)_{i, j=1, \ldots, d}
\end{aligned}
$$

By [3, theorem 6.15], $\operatorname{deg}\left(\phi_{L}\right)=\operatorname{deg}(\mu)^{2}$, and by the Riemann-Roch theorem $[\mathbf{1 4}, \S 16],\left(\operatorname{deg}_{L}(A) / d !\right)^{2}=\operatorname{deg}\left(\phi_{L}\right)$. Since $L$ is ample, $\operatorname{deg}_{L}(A)>$ 0 and hence equals $\operatorname{covol}(\Lambda) H_{q}$.

q.e.d.

2.3. Toric metrized line bundles. Recall that an element $f$ inside $\mathscr{C}_{\text {rpoly }}\left(\mathbb{R}^{d}\right)$ is a continuous real function on $\mathbb{R}^{d}$ such that there is a rational polytopal decomposition $\mathcal{C}$ of $\mathbb{R}^{d}$ satisfying that $f$ restricted to all $\Delta \in \mathcal{C}$ is affine and takes rational values on vertices. The following proposition is due to Gubler. 
Proposition 2.2. Let $L=\left(T \times \mathbb{G}_{a, k}\right) / M$ be a line bundle on $A$ given by a cocycle $\left(Z_{\mu}\right)_{\mu \in M}$ as above. Let $\mathcal{A}$ be a formal $k^{\circ}$-model determined by a rational polytopal decomposition $\mathcal{C}_{\Lambda}$ of $\mathbb{R}^{d} / \Lambda$ given by $\mathcal{C}$.

1) There is a one-to-one correspondence between the following two sets: The first consists of all formal metrics of L, i.e., formal model $\mathcal{L}$ of $L^{e}$ (with e minimal) on $\mathcal{A}$, with trivialization $\left(\mathcal{U}_{\Delta}\right)_{\Delta \in \mathcal{C}_{\Lambda}}$; and the second consists of functions $g \in \mathscr{C}_{\text {rpoly }}\left(\mathbb{R}^{d}\right)$, affine on $\Delta$ for any $\Delta \in \mathcal{C}$, satisfying

$$
g(\mathbf{x}+\boldsymbol{\lambda})=g(\mathbf{x})+z_{\boldsymbol{\lambda}}(\mathbf{x}) ; \quad \boldsymbol{\lambda} \in \Lambda, \mathbf{x} \in \mathbb{R}^{d} .
$$

Moreover, if we denote by \|\| the corresponding formal metric on $L$, then we have

$$
g \circ \tau=-\log \left(p^{*}\|1\|\right)
$$

on $T^{\mathrm{an}}$, where $p: T^{\mathrm{an}} \rightarrow A^{\text {an }}$ is the natural projection and $p^{*}\|\cdot\|$ is the pull-back metric on the trivial line bundle on $T^{\mathrm{an}}$.

2) The reduction $\widetilde{\mathcal{L}}$ is ample if and only if $g$ is strongly polytopal convex with respect to $\mathcal{C}$.

Proof. For (1), by (2.4) and the fact that there exists $\mathbf{m}_{\boldsymbol{\lambda}} \in \mathbb{Z}^{d}$ for all $\boldsymbol{\lambda} \in \Lambda$ such that $b(\mathbf{x}, \boldsymbol{\lambda})=\mathbf{m}_{\boldsymbol{\lambda}} \cdot \mathbf{x}$, we can find a smallest integer $e \geq 1$ such that $e \cdot g$ has integer gradient everywhere. Then by $[\mathbf{1 1}$, proposition 6.6], $e \cdot g$ determines a formal model $\mathcal{L}$ of $L^{e}$. Hence $g$ determines a formal metric on $L$. The last identity (2.5) follows from the same proposition. For (2), it follows from [11, corollary 6.7]. q.e.d.

Definition 2.3. Given a class $L \in \operatorname{Pic}(A) \otimes_{\mathbb{Z}} \mathbb{Q}$ such that $L^{e}$ is a line bundle, we call a metric on $L$ whose $e$-th power is a metric on $L^{e}$ determined by the above proposition a toric formal metric, and the corresponding function $g$ (divided by $e$ ) the associated formal Green function. We denote by $\mathscr{G}_{\text {for }}(L)$ the set of all formal Green functions of $L$, and $\mathscr{G}_{+}(L)$ the set of all uniform limits of formal Green functions of $L$ associated to semipositive toric formal metrics which we call semipositive Green functions. It is easy to see that $g \in \mathscr{G}_{+}(L)$ also satisfies (2.4), and the metric determined by (2.5) is semipositive in its original sense. Similarly, we denote by $\mathscr{G}_{\text {int }}(L)$ the set of difference of functions in $\mathscr{G}_{+}\left(L^{\prime}\right)$ and $\mathscr{G}_{+}\left(L^{\prime \prime}\right)$ with $L=L^{\prime} \otimes\left(L^{\prime \prime}\right)^{-1}$ which we call integrable Green functions; hence the corresponding metric is integrable. Moreover, the set of integrable Green functions for all classes $L \in \operatorname{Pic}(A) \otimes_{\mathbb{Z}} \mathbb{Q}$ : $\mathscr{G}_{\text {int }}(A)=\bigcup \mathscr{G}_{\text {int }}(L)$ is a vector space over $\mathbb{Q}$. All classes in $\operatorname{Pic}(A) \otimes_{\mathbb{Z}} \mathbb{Q}$ equipped with a metric corresponding to a Green function in $\mathscr{G}_{\text {int }}(A)$ are called toric metrized line bundles. At last we denote by $\mathscr{G}(L)$ the set 
of continuous real functions satisfying (2.4), and we simply call them Green functions of $L$.

The following theorem provides a certain large class of semipositive Green functions for an ample line bundle.

Theorem 2.4. Let $L \in \operatorname{Pic}(A) \otimes_{\mathbb{Z}} \mathbb{Q}$ be an ample class and $g \in \mathscr{G}(L) \cap$ $\mathscr{C}^{2}\left(\mathbb{R}^{d}\right)$ such that the Hessian matrix $\left(g_{i j}(\mathbf{x})\right)_{i, j=1, \ldots, d}$ is semipositive definite everywhere; then $g$ is inside $\mathscr{G}_{+}(L)$.

Remark 2.5. This theorem and its Corollary 2.11 are crucial for our main results since they provide a large class of functions which should be the Green functions of toric metrics on $L$. The proof is based on Lemmas 2.6 to 2.10, in which Lemma 2.9 will also be used in the proof of Theorem 3.3. But we will not single it out, since it appears naturally in a series of estimates, as we will see in the following proof.

Proof. The proof is divided into several steps.

Step 1. We reduce to the case where $\left(g_{i j}(\mathbf{x})\right)_{i, j=1, \ldots, d}$ is positive definite for all $\mathbf{x} \in \mathbb{R}^{d}$.

First, there exists a function $g_{\text {can }} \in \mathscr{G}(L) \cap \mathscr{C}^{2}\left(\mathbb{R}^{d}\right)$ such that the matrix $\left(\left(g_{\text {can }}\right)_{i j}(\mathbf{x})\right)$ is positive definite. Indeed, by [8, lemma 6.5.2 (4)], there is a group homomorphism $c: \Lambda \rightarrow \mathbb{Q}$ such that $z_{\boldsymbol{\lambda}}(\mathbf{0})=q(\boldsymbol{\lambda})+c(\boldsymbol{\lambda})$. We linearly extend $c$ to $\mathbb{R}^{d}$ and define $g_{\text {can }}=q+c$; then $g_{\text {can }} \in \mathscr{G}(L) \cap$ $\mathscr{C}^{2}\left(\mathbb{R}^{d}\right)$ and $\left(\left(g_{\text {can }}\right)_{i j}(\mathbf{x})\right)$ is a constant positive definite matrix. Next, for any $g \in \mathscr{G}(L) \cap \mathscr{C}^{2}\left(\mathbb{R}^{d}\right), g_{i j}$ is $\Lambda$-periodic for any $(i, j)$ and $g-g_{\text {can }}$ is a $\Lambda$-periodic $\mathscr{C}^{2}$-function. For any $g$ in the proposition, let $f=g-g_{\text {can }}$ and $g_{t}=g_{\text {can }}+t f$ for $t \in[0,1]$; then $g_{t} \rightarrow g_{1}=g$ when $t \rightarrow 1$ and $\left(\left(g_{t}\right)_{i j}(\mathbf{x})\right)$ are positive definite for all $t<1$. The claim follows.

Step 2. Now fix a function $g$ as in the proposition, but with the condition that $\left(g_{i j}(\mathbf{x})\right)_{i, j=1, \ldots, d}$ is positive definite; we are going to construct a sequence of functions $g_{n} \in \mathscr{G}_{\text {for }}(L) \cap \mathscr{G}_{+}(L)$ approaching $g$. For any $\mathbf{u} \in \mathbb{S}^{d-1}$, the function $\nabla_{\mathbf{u}} \nabla_{\mathbf{u}} g$ is $\Lambda$-periodic and strictly positive; hence there exist $0<h_{g}<H_{g}$ such that $h_{g}<\nabla_{\mathbf{u}} \nabla_{\mathbf{u}} g(\mathbf{x})<H_{g}$ for any $\mathbf{u} \in \mathbb{S}^{d-1}$ and $\mathbf{x} \in \mathbb{R}^{d}$.

Let $N$ be a sufficiently large integer; for $\mathbf{j}=\left(j_{1}, \ldots, j_{d}\right) \in\left(\frac{1}{N} \mathbb{Z}\right)^{d}$, we let $\boldsymbol{\lambda}_{\mathbf{j}}=j_{1} \boldsymbol{\lambda}_{1}+\cdots+j_{d} \boldsymbol{\lambda}_{d}$. For each $\mathbf{j}$ such that $\boldsymbol{\lambda}_{\mathbf{j}} \in \mathfrak{F}$, we choose a positive number $\epsilon_{N}(\mathbf{j})<\frac{1}{N^{2}}$ such that $g\left(\boldsymbol{\lambda}_{\mathbf{j}}\right)-\epsilon_{N}(\mathbf{j}) \in \mathbb{Q}$ and a vector $\boldsymbol{\epsilon}_{N}(\mathbf{j})$ such that $\left\|\boldsymbol{\epsilon}_{N}(\mathbf{j})\right\|<\frac{1}{N}, \nabla g\left(\boldsymbol{\lambda}_{\mathbf{j}}\right)-\boldsymbol{\epsilon}_{N}(\mathbf{j}) \in \mathbb{Q}^{d}$ and the graph of the function

$$
g_{\boldsymbol{\lambda}_{\mathbf{j}}}^{(N)}(\mathbf{x})=\left(\nabla g\left(\boldsymbol{\lambda}_{\mathbf{j}}\right)-\boldsymbol{\epsilon}_{N}(\mathbf{j})\right)\left(\mathbf{x}-\boldsymbol{\lambda}_{\mathbf{j}}\right)+g\left(\boldsymbol{\lambda}_{\mathbf{j}}\right)-\epsilon_{N}(\mathbf{j})
$$


is below the graph of $g$, which is possible since $g$ is strictly convex. For general $\mathbf{j}$, we let $\mathbf{j}_{0}$ be the unique element in $\left(\frac{1}{N} \mathbb{Z}\right)^{d}$ such that $\mathbf{j}-\mathbf{j}_{0} \in \mathbb{Z}^{d}$ and $\lambda_{\mathbf{j}_{0}} \in \mathfrak{F}$. Then we define

$$
g_{\boldsymbol{\lambda}_{\mathbf{j}}}^{(N)}(\mathbf{x})=\left(\nabla g\left(\boldsymbol{\lambda}_{\mathbf{j}}\right)-\boldsymbol{\epsilon}_{N}\left(\mathbf{j}_{0}\right)\right)\left(\mathbf{x}-\boldsymbol{\lambda}_{\mathbf{j}}\right)+g\left(\boldsymbol{\lambda}_{\mathbf{j}}\right)-\epsilon_{N}\left(\mathbf{j}_{0}\right)
$$

By construction, $g_{\boldsymbol{\lambda}_{\mathbf{j}}}^{(N)} \in \mathscr{C}_{\text {rpoly }}(\mathbb{R})$. We need the following lemma.

Lemma 2.6. For each $\mathbf{j} \in\left(\frac{1}{N} \mathbb{Z}\right)^{d}$ and $\boldsymbol{\lambda} \in \Lambda$, we have

$$
g(\mathbf{x})-g_{\boldsymbol{\lambda}_{\mathbf{j}}}^{(N)}(\mathbf{x})=g(\mathbf{x}+\boldsymbol{\lambda})-g_{\boldsymbol{\lambda}_{\mathbf{j}}+\boldsymbol{\lambda}}^{(N)}(\mathbf{x}+\boldsymbol{\lambda}) .
$$

Proof. By definition, we have

$$
\begin{aligned}
& g_{\boldsymbol{\lambda}_{\mathbf{j}}+\boldsymbol{\lambda}}^{(N)}(\mathbf{x}+\boldsymbol{\lambda})-g_{\boldsymbol{\lambda}_{\mathbf{j}}}^{(N)}(\mathbf{x}) \\
= & \left(\nabla g\left(\boldsymbol{\lambda}_{\mathbf{j}}+\boldsymbol{\lambda}\right)-\nabla g\left(\boldsymbol{\lambda}_{\mathbf{j}}\right)\right)\left(\mathbf{x}-\boldsymbol{\lambda}_{\mathbf{j}}\right)+g\left(\boldsymbol{\lambda}_{\mathbf{j}}+\boldsymbol{\lambda}\right)-g\left(\boldsymbol{\lambda}_{\mathbf{j}}\right) \\
= & \nabla z_{\boldsymbol{\lambda}}\left(\boldsymbol{\lambda}_{\mathbf{j}}\right)\left(\mathbf{x}-\boldsymbol{\lambda}_{\mathbf{j}}\right)+z_{\boldsymbol{\lambda}}\left(\boldsymbol{\lambda}_{\mathbf{j}}\right) \\
= & z_{\boldsymbol{\lambda}}(\mathbf{x}) \\
= & g(\mathbf{x}+\boldsymbol{\lambda})-g(\mathbf{x})
\end{aligned}
$$

where the third equality is because $z_{\lambda}$ is affine.

q.e.d.

Step 3. We define a function $g^{(N)}$ by

$$
g^{(N)}(\mathbf{x})=\sup _{\mathbf{j}} g_{\boldsymbol{\lambda}_{\mathbf{j}}}^{(N)}(\mathbf{x})
$$

which is less than $g(\mathbf{x})$. We have

Lemma 2.7. For any compact subset $V \subset \mathbb{R}^{d}$, there exists a finite subset $\mathcal{J}_{V} \subset\left(\frac{1}{N} \mathbb{Z}\right)^{d}$ such that

$$
g^{(N)}(\mathbf{x})=\max _{\mathbf{j} \in \mathcal{J}_{V}} g_{\mathbf{\lambda}_{\mathbf{j}}}^{(N)}(\mathbf{x})
$$

for all $\mathbf{x} \in V$.

Proof. We only need to prove that for given $M \in \mathbb{R}$, there are only finitely many $\mathbf{j}$ such that $g_{\boldsymbol{\lambda}_{\mathbf{j}}}^{(N)}(\mathbf{x}) \geq M$ for some $\mathbf{x} \in V$. For a given $\mathbf{j}$, we try to give a lower bound for the difference $g(\mathbf{x})-g_{\boldsymbol{\lambda}_{\mathbf{j}}}^{(N)}(\mathbf{x})$. Let 


$$
\begin{aligned}
\mathbf{u}= & \mathbf{x}-\boldsymbol{\lambda}_{\mathbf{j}} ; \text { by definition, } \\
& g(\mathbf{x})-g_{\boldsymbol{\lambda}_{\mathbf{j}}}^{(N)}(\mathbf{x}) \\
> & \left(g(\mathbf{x})-g_{\boldsymbol{\lambda}_{\mathbf{j}}}^{(N)}(\mathbf{x})\right)-\left(g\left(\boldsymbol{\lambda}_{\mathbf{j}}\right)-g_{\boldsymbol{\lambda}_{\mathbf{j}}}^{(N)}\left(\boldsymbol{\lambda}_{\mathbf{j}}\right)\right) \\
= & \int_{0}^{1} \frac{\mathrm{d}}{\mathrm{d} t}\left(g\left(\boldsymbol{\lambda}_{\mathbf{j}}+t \mathbf{u}\right)-g_{\boldsymbol{\lambda}_{\mathbf{j}}}^{(N)}\left(\boldsymbol{\lambda}_{\mathbf{j}}+t \mathbf{u}\right)\right) \mathrm{d} t \\
= & \int_{0}^{1} \nabla_{\mathbf{u}} g\left(\boldsymbol{\lambda}_{\mathbf{j}}+t \mathbf{u}\right) \mathrm{d} t-\nabla_{\mathbf{u}} g\left(\boldsymbol{\lambda}_{\mathbf{j}}\right)+\boldsymbol{\epsilon}_{N}\left(\mathbf{j}_{0}\right) \cdot \mathbf{u} \\
= & \int_{0}^{1}\left(\nabla_{\mathbf{u}} g\left(\boldsymbol{\lambda}_{\mathbf{j}}\right)+\int_{0}^{t} \nabla_{\mathbf{u}} \nabla_{\mathbf{u}} g\left(\boldsymbol{\lambda}_{\mathbf{j}}+s \mathbf{u}\right) \mathrm{d} s\right) \mathrm{d} t-\nabla_{\mathbf{u}} g\left(\boldsymbol{\lambda}_{\mathbf{j}}\right)+\boldsymbol{\epsilon}_{N}\left(\mathbf{j}_{0}\right) \cdot \mathbf{u} \\
= & \int_{0}^{1} \int_{0}^{t} \nabla_{\mathbf{u}} \nabla_{\mathbf{u}} g\left(\boldsymbol{\lambda}_{\mathbf{j}}+s \mathbf{u}\right) \mathrm{d} s \mathrm{~d} t+\boldsymbol{\epsilon}_{N}\left(\mathbf{j}_{0}\right) \cdot \mathbf{u} \\
> & \frac{h_{g}\|\mathbf{u}\|^{2}}{2}-\left\|\boldsymbol{\epsilon}_{N}\left(\mathbf{j}_{0}\right)\right\| \cdot\|\mathbf{u}\| \\
> & \frac{h_{g}\|\mathbf{u}\|^{2}}{2}-\frac{\|\mathbf{u}\|}{N} .
\end{aligned}
$$

We see that there is $N_{M}>0$ such that $\left\|\lambda_{\mathbf{j}}-\mathbf{x}\right\|>N_{M}$ implies $g_{\boldsymbol{\lambda}_{\mathbf{j}}}^{(N)}(\mathbf{x})<M$. Hence the lemma follows.

The above lemma implies that $g^{(N)} \in \mathscr{C}_{\text {rpoly }}\left(\mathbb{R}^{d}\right)$ and is convex. On the other hand, if $g^{(N)}(\mathbf{x})=g_{\boldsymbol{\lambda}_{\mathbf{j}}}^{(N)}(\mathbf{x})$ for some $\mathbf{j}$, then $g^{(N)}(\mathbf{x}+\boldsymbol{\lambda})=$ $g_{\boldsymbol{\lambda}_{\mathbf{j}}+\boldsymbol{\lambda}}^{(N)}(\mathbf{x}+\boldsymbol{\lambda})$ for all $\boldsymbol{\lambda} \in \Lambda$ since, by Lemma $2.6, g_{\boldsymbol{\lambda}_{\mathbf{j}^{\prime}}}^{(N)}(\mathbf{x}+\boldsymbol{\lambda})>g_{\boldsymbol{\lambda}_{\mathbf{j}}+\boldsymbol{\lambda}}^{(N)}(\mathbf{x}+\boldsymbol{\lambda})$ will imply that $g_{\boldsymbol{\lambda}_{\mathbf{j}^{\prime}-\boldsymbol{\lambda}}}^{(N)}(\mathbf{x})>g_{\boldsymbol{\lambda}_{\mathbf{j}}}^{(N)}(\mathbf{x})$, which is a contradiction. Again by the same lemma, we conclude that $g^{(N)}$ satisfies (2.4) and hence is inside $\mathscr{G}(L)$.

Step 4. Before proving that $g^{(N)}$ is semipositive formal, we would like to bound the difference of it and $g$.

Lemma 2.8. For any $\mathrm{x} \in \mathbb{R}^{d}$, we have

$$
0 \leq g(\mathbf{x})-g^{(N)}(\mathbf{x})<\frac{R_{\mathfrak{F}}^{2} \cdot H_{g}+2 R_{\mathfrak{F}}+2}{2 N^{2}} .
$$

Proof. The proof follows the same line as in Lemma 2.7. Hence we have

$$
g(\mathbf{x})-g^{(N)}(\mathbf{x})<\frac{H_{g}}{2}\left\|\boldsymbol{\lambda}_{\mathbf{j}}-\mathbf{x}\right\|^{2}+\frac{1}{N}\left\|\boldsymbol{\lambda}_{\mathbf{j}}-\mathbf{x}\right\|+\frac{1}{N^{2}}
$$

for any $\mathbf{j}$. In fact, we can choose $\mathbf{j}$ such that $\left\|\boldsymbol{\lambda}_{\mathbf{j}}-\mathbf{x}\right\| \leq \frac{R_{\mathfrak{F}}}{N}$. Hence the lemma follows.

q.e.d.

Conversely, we have the following lemma on the estimate of the gradient, which will be used also in the next section. 
Lemma 2.9. Let $f$ be any convex rational polytopal continuous function on $\mathbb{R}^{d}$. Suppose that $|f(\mathbf{x})-g(\mathbf{x})|<\epsilon$ for any $\mathbf{x} \in \mathbb{R}^{d}$; then for any d-dimensional polytope $\Delta$ on which $f$ is affine, $\left\|\mathbf{m}_{\Delta}-\nabla g\left(\mathbf{x}_{0}\right)\right\| \leq$ $2 \sqrt{\epsilon H_{g}}$ for all $\mathbf{x}_{0} \in \Delta$, where $\mathbf{m}_{\Delta}$ is the gradient of $f$ on $\Delta$.

Proof. By continuity, we can assume that $\mathbf{x}_{0} \in \operatorname{int}(\Delta)$ and $\mathbf{x}_{0}=\mathbf{0}$. We only need to prove the lemma for $\tilde{f}$ and $\tilde{g}$ where $\tilde{f}(\mathbf{x})=f(\mathbf{x})-$ $\nabla g(\mathbf{0}) \cdot(\mathbf{x})-g(\mathbf{0})$ and $\tilde{g}(\mathbf{x})=g(\mathbf{x})-\nabla g(\mathbf{0}) \cdot(\mathbf{x})-g(\mathbf{0})$. In this case, we need to prove that $\left\|\mathbf{m}_{\Delta}\right\|<2 \sqrt{\epsilon H_{g}}$. For any $\mathbf{u} \in \mathbb{S}^{d-1}$, we assume that $\mathbf{m}_{\Delta} \cdot \mathbf{u} \geq 0$, since otherwise we can take $-\mathbf{u}$. For $t>0$, consider

$$
\begin{aligned}
& (\tilde{f}(t \mathbf{u})-\tilde{g}(t \mathbf{u}))-(\tilde{f}(\mathbf{0})-\tilde{g}(\mathbf{0})) \\
= & \int_{0}^{t}\left(\nabla_{\mathbf{u}} \tilde{f}(s \mathbf{u})-\nabla_{\mathbf{u}} \tilde{g}(s \mathbf{u})\right) \mathrm{d} s \\
\geq & \mathbf{m}_{\Delta} \cdot \mathbf{u} t-\int_{0}^{t} \int_{0}^{s} \nabla_{\mathbf{u}} \nabla_{\mathbf{u}} \tilde{g}(r \mathbf{u}) \mathrm{d} r \mathrm{~d} s \\
> & \mathbf{m}_{\Delta} \cdot \mathbf{u} t-\frac{H_{g} t^{2}}{2}
\end{aligned}
$$

where the first inequality is due to the assumption that $f$ is convex. On the other hand, it is less than $2 \epsilon$; hence we have

$$
\frac{H_{g} t^{2}}{2}-\mathbf{m}_{\Delta} \cdot \mathbf{u} t+2 \epsilon>0
$$

for all $t>0$. Hence $\mathbf{m}_{\Delta} \cdot \mathbf{u}<2 \sqrt{\epsilon H_{g}}$ and then $\left\|\mathbf{m}_{\Delta}\right\| \leq 2 \sqrt{\epsilon H_{g}}$. q.e.d.

The above lemma immediately implies the following:

Lemma 2.10. Let $f$ and $\Delta$ be as above; then for any $\mathbf{x}, \mathbf{x}^{\prime} \in \Delta$, the distance $\left\|\mathbf{x}-\mathbf{x}^{\prime}\right\| \leq \frac{4}{h_{g}} \sqrt{\epsilon H_{g}}$.

Proof. Let $\mathbf{x}^{\prime}-\mathbf{x}=t \mathbf{u}$ with $\mathbf{u} \in \mathbb{S}^{d-1}$ and $t=\left\|\mathbf{x}-\mathbf{x}^{\prime}\right\| \geq 0$. Since $\mathbf{x}, \mathbf{x}^{\prime} \in \Delta$ and $f$ is affine on $\Delta$, we have

$$
h_{g} t \leq\left|\int_{0}^{t} \nabla_{\mathbf{u}} \nabla_{\mathbf{u}} g(\mathbf{x}+s \mathbf{u}) \mathrm{d} s\right|=\left|\nabla_{\mathbf{u}} g\left(\mathbf{x}^{\prime}\right)-\nabla_{\mathbf{u}} g(\mathbf{x})\right| \leq 4 \sqrt{\epsilon H_{g}}
$$

by the above lemma. Hence $t=\left\|\mathbf{x}-\mathbf{x}^{\prime}\right\| \leq \frac{4}{h_{g}} \sqrt{\epsilon H_{g}}$.

q.e.d.

In particular, if we apply the above lemma to $g^{(N)}$, we see that $\Delta$ is compact for any maximal connected subset $\Delta$ on which $g^{(N)}$ is affine.

Step 5. We would like to apply Lemma 1.1. Hence we need to show that $\Delta$ is convex. By Lemma 2.7, $g^{(N)}$ coincides with some $g_{\boldsymbol{\lambda}_{\mathbf{j}}}^{(N)}$ restricted to $\Delta$. Suppose that there are $\mathbf{x}_{0}, \mathbf{x}_{1} \in \Delta$ and $t \in(0,1)$ such that $\mathbf{x}_{t}=t \mathbf{x}_{1}+(1-t) \mathbf{x}_{0} \notin \Delta$; then $g^{(N)}\left(\mathbf{x}_{t}\right)=g_{\boldsymbol{\lambda}_{\mathbf{j}^{\prime}}}^{(N)}\left(\mathbf{x}_{t}\right)$ for some $\mathbf{j}^{\prime} \neq \mathbf{j}$. Again by construction, $g_{\boldsymbol{\lambda}_{\mathbf{j}^{\prime}}}^{(N)}\left(\mathbf{x}_{t}\right)>g_{\boldsymbol{\lambda}_{\mathbf{j}}}^{(N)}\left(\mathbf{x}_{t}\right)$. Hence there is one point 
$\mathbf{x} \in\left\{\mathbf{x}_{0}, \mathbf{x}_{1}\right\}$ such that $g_{\boldsymbol{\lambda}_{\mathbf{j}^{\prime}}}^{(N)}(\mathbf{x})>g_{\boldsymbol{\lambda}_{\mathbf{j}}}^{(N)}(\mathbf{x})$ which is a contradiction. Now by Lemma 1.1, $g^{(N)}$ determines a $\Lambda$-periodic rational polytopal decomposition $\mathcal{C}$ of $\mathbb{R}^{d}$.

Finally, we prove that for any $\Delta \in \mathcal{C}, \Delta$ maps bijectively to its image under the projection $p: \mathbb{R}^{d} \rightarrow \mathbb{R}^{d} / \Lambda$ when $N$ is sufficiently large. We can $\operatorname{assume} \operatorname{dim}(\Delta)=d$. By Lemmas 2.8 and 2.10, we see that this holds if

$$
\frac{4}{h_{g}} \sqrt{\frac{R_{\mathfrak{F}}^{2} \cdot H_{g}+2 R_{\mathfrak{F}}+2}{2 N^{2}} \cdot H_{g}}<2 r_{\mathfrak{F}} .
$$

Now by Proposition 2.2, $g^{(N)}$ is a semipositive formal Green function for large $N$. Hence the theorem follows by Lemma 2.8 .

The above theorem has the following direct corollary.

Corollary 2.11. For any class $L \in \operatorname{Pic}(A) \otimes_{\mathbb{Z}} \mathbb{Q}$, if $g \in \mathscr{G}(L) \cap$ $\mathscr{C}^{2}\left(\mathbb{R}^{d}\right)$, then $g$ is integrable, i.e., $g \in \mathscr{G}_{\text {int }}(L)$.

\section{A Limit Formula for the Measure}

In this section, we prove a formula for the measure of metrics determined by certain integrable Green functions.

3.1. Measures on torus and mixed Hessian. Recall that we have a closed manifold $\mathbb{R}^{d} / \Lambda$. Similar to $\mathbb{R}^{d}$ as in 2.2 , we define various spaces of real functions $\mathscr{C}^{?}\left(\mathbb{R}^{d} / \Lambda\right), \mathscr{C}_{>0}^{?}\left(\mathbb{R}^{d} / \Lambda\right)$, and $\mathscr{C}_{>0}^{?}\left(\mathbb{R}^{d} / \Lambda\right)$ for $?=l ; \infty ; k, \alpha$. A measure on $\mathbb{R}^{d} / \Lambda$ is a continuous linear functional $\mu: \mathscr{C}\left(\mathbb{R}^{d} / \Lambda\right) \rightarrow \mathbb{R} ;$ it is semipositive if $\mu(f) \geq 0$ for all $f \in \mathscr{C}_{\geq 0}\left(\mathbb{R}^{d} / \Lambda\right)$; positive if $\mu(f)>0$ for $0 \neq f \in \mathscr{C}_{\geq 0}\left(\mathbb{R}^{d} / \Lambda\right)$. The space of all (resp. semipositive, positive) measures is denoted by $\mathscr{M}\left(\mathbb{R}^{d} / \Lambda\right)\left(\right.$ resp. $\mathscr{M}_{\geq 0}\left(\mathbb{R}^{d} / \Lambda\right)$, $\left.\mathscr{M}_{>0}\left(\mathbb{R}^{d} / \Lambda\right)\right)$. It is endowed with the weak-* topology; i.e., a sequence $\mu_{n} \rightarrow \mu$ if and only if $\mu_{n}(f) \rightarrow \mu(f)$ for all $f \in \mathscr{C}\left(\mathbb{R}^{d} / \Lambda\right)$. Recall that we have the Lebesgue measure $\mathrm{d} \mathbf{x}$ on $\mathbb{R}^{d} / \Lambda$; hence the spaces of functions $\mathscr{C}^{?}\left(\mathbb{R}^{d} / \Lambda\right)$ can be identified as subspaces $\mathscr{M}^{?}\left(\mathbb{R}^{d} / \Lambda\right)$ of the space of measures by integration against $\mathrm{d} \mathbf{x}$ for $?=l ; \infty ; k, \alpha$. Under this identification, being semipositive (i.e., non-negative) or positive (i.e., strictly positive) for a function coincides with that for a measure. Hence we also introduce the notation $\mathscr{M}_{\geq 0}^{?}\left(\mathbb{R}^{d} / \Lambda\right)$ or $\mathscr{M}_{>0}^{?}\left(\mathbb{R}^{d} / \Lambda\right)$ for $?=l ; \infty ; k, \alpha$ for their obvious meaning. We write $\mu \leq \mu^{\prime}$ if $\mu^{\prime}-\mu \in \mathscr{M}_{\geq 0}\left(\mathbb{R}^{d} / \Lambda\right)$. Finally, we denote by $|\mu|$ the total mass of $\mu$, i.e., $|\mu|=\mu(1)$. It is easy to see that if $\mu$ is semipositive and $|\mu|=0$, then $\mu=0$. 
Definition 3.1. For functions $g_{1}, \ldots, g_{d} \in \mathscr{C}^{2}\left(\mathbb{R}^{d}\right)$, we define the so-called mixed Hessian of $g_{1}, \ldots, g_{d}$ to be the function

$$
\operatorname{Hess}_{g_{1}, \ldots, g_{d}}(\mathbf{x})=\sum_{\mu, \nu \in \mathfrak{S}_{d}} \epsilon(\mu \nu) \prod_{i=1}^{d}\left(g_{i}\right)_{\mu(i) \nu(i)}(\mathbf{x})
$$

where $\epsilon(\mu)=1$ (resp. -1) if $\mu$ is an even (resp. odd) permutation. (The subscript $i$ in $g_{i}$ is not to be confused with the one for the $i$-th derivative of $g$.) In particular, when $g_{1}=\cdots=g_{d}=g$,

$$
\operatorname{Hess}_{g}(\mathbf{x}):=\operatorname{Hess}_{g, \ldots, g}(\mathbf{x})=d ! \operatorname{det}\left(g_{i j}(\mathbf{x})\right)_{i, j=1, \ldots, d}
$$

is $d$ ! times the determinant of the usual Hessian matrix of $g$.

The following lemma is an easy exercise in calculus.

Lemma 3.2. Let $L \in \operatorname{Pic}(A) \otimes_{\mathbb{Z}} \mathbb{Q}$ be an ample class and $g_{1}, \ldots, g_{d} \in$ $\mathscr{G}(L) \cap \mathscr{C}^{3}\left(\mathbb{R}^{d}\right)$; then $\operatorname{Hess}_{g_{1}, \ldots, g_{d}}$ is $\Lambda$-periodic and hence in $\mathscr{C}^{1}\left(\mathbb{R}^{d} / \Lambda\right)$. We have

$$
\int_{\mathbb{R}^{d} / \Lambda} \operatorname{Hess}_{g_{1}, \ldots, g_{d}}(\mathbf{x}) \mathrm{d} \mathbf{x}=\operatorname{deg}_{L}(A) .
$$

Proof. First, we check the case $g_{1}=\cdots=g_{d}=g_{\text {can }}$. Then

$$
\int_{\mathbb{R}^{d} / \Lambda} \operatorname{Hess}_{g_{\text {can }}}(\mathbf{x}) \mathrm{d} \mathbf{x}=\int_{\mathbb{R}^{d} / \Lambda} H_{q} \mathrm{~d} \mathbf{x}=\operatorname{covol}(\Lambda) H_{q}
$$

which equals $\operatorname{deg}_{L}(A)$ by Lemma 2.1. In general, since any two $g, g^{\prime} \in$ $\mathscr{G}(L) \cap \mathscr{C}^{3}\left(\mathbb{R}^{d}\right)$ differ by a $\Lambda$-periodic function $f \in \mathscr{C}^{3}\left(\mathbb{R}^{d}\right)$, by multilinearity and symmetry, we only need to prove that for such $f$ and $g_{2}, \ldots, g_{d} \in \mathscr{G}(L) \cap \mathscr{C}^{3}\left(\mathbb{R}^{d}\right)$,

$$
\int_{\mathbb{R}^{d} / \Lambda} \operatorname{Hess}_{f, g_{2}, \ldots, g_{d}}(\mathbf{x}) \mathrm{d} \mathbf{x}=0 .
$$

By a linear change of coordinates, one can assume that $\boldsymbol{\lambda}_{i}=\mathbf{e}_{i}$ for $i=1, \ldots, d$. Hence $(3.1)$ becomes

$$
\int_{[0,1]^{d}} \operatorname{Hess}_{f, g_{2}, \ldots, g_{d}}(\mathbf{x}) \mathrm{d} x_{1} \cdots \mathrm{d} x_{d}=0 .
$$

For each $i=1, \ldots, d$, let us denote by

$$
\begin{aligned}
& F_{i}^{+}=\left\{\mathbf{x}=\left(x_{1}, \ldots, x_{d}\right) \in[0,1]^{d} \mid x_{i}=1\right\} \\
& F_{i}^{-}=\left\{\mathbf{x}=\left(x_{1}, \ldots, x_{d}\right) \in[0,1]^{d} \mid x_{i}=0\right\} .
\end{aligned}
$$


Then

$$
\begin{aligned}
& \int_{[0,1]^{d}} \operatorname{Hess}_{f, g_{2}, \ldots, g_{d}}(\mathbf{x}) \mathrm{d} x_{1} \cdots \mathrm{d} x_{d} \\
= & \int_{[0,1]^{d}} \sum_{\mu, \nu \in \mathfrak{S}_{d}} \epsilon(\mu \nu) f_{\mu(1) \nu(1)} \prod_{i=2}^{d}\left(g_{i}\right)_{\mu(i) \nu(i)} \mathrm{d} x_{1} \cdots \mathrm{d} x_{d} \\
= & \sum_{\mu, \nu \in \mathfrak{S}_{d}} \epsilon(\mu \nu) \\
& \left(\int_{F_{\mu(1)}^{+}} f_{\nu(1)} \prod_{i=2}^{d}\left(g_{i}\right)_{\mu(i) \nu(i)} \mathrm{d} \mathbf{y}-\int_{F_{\mu(1)}^{-}} f_{\nu(1)} \prod_{i=2}^{d}\left(g_{i}\right)_{\mu(i) \nu(i)} \mathrm{d} \mathbf{y}-S_{\mu, \nu}\right) \\
= & -\sum_{\mu, \nu \in \mathfrak{S}_{d}} \epsilon(\mu \nu) S_{\mu, \nu}
\end{aligned}
$$

since $f$ and $\left(g_{i}\right)_{\mu(i) \nu(i)}$ are $\Lambda$-periodic, where

$$
S_{\mu, \nu}=\int_{[0,1]^{d}} f_{\nu(1)} \sum_{j=2}^{d}\left(g_{j}\right)_{\mu(1) \mu(j) \nu(j)} \prod_{\substack{i=2 \\ i \neq j}}^{d}\left(g_{i}\right)_{\mu(i) \nu(i)} \mathrm{d} x_{1} \cdots \mathrm{d} x_{d} .
$$

But then

$$
\begin{aligned}
& \sum_{\mu, \nu \in \mathfrak{S}_{d}} \epsilon(\mu \nu) S_{\mu, \nu} \\
= & \sum_{j=2}^{d} \sum_{\nu \in \mathfrak{S}_{d}} \epsilon(\nu) \\
& \int_{[0,1]^{d}} f_{\nu(1)}\left(\sum_{\mu \in \mathfrak{S}_{d}} \epsilon(\mu)\left(g_{j}\right)_{\mu(1) \mu(j) \nu(j)} \prod_{\substack{i=2 \\
i \neq j}}^{d}\left(g_{i}\right)_{\mu(i) \nu(i)}\right) \mathrm{d} x_{1} \cdots \mathrm{d} x_{d} \\
= & 0
\end{aligned}
$$

since in the inner summation, the terms with $\mu$ and $\mu^{\prime}$ such that $\mu^{\prime}(1)=$ $\mu(j), \mu^{\prime}(j)=\mu(1)$, and $\mu^{\prime}(i)=\mu(i)$ for $i \neq 1, j$ cancel each other. Hence the lemma follows.

q.e.d.

3.2. Limit formula for the measure. Before we state the formula, we would like to introduce the notion of dual polytopes. The main reference for this is $[\mathbf{1 3}, \S 2, \S 3]$. Let $\mathcal{C}$ be a (rational) polytopal decomposition of $\mathbb{R}^{d}$ and $f$ be a (rational) polytopal function, strongly polytopal convex with respect to $\mathcal{C}$. Then for any vertex $\mathbf{v} \in \mathcal{C}$, let $\operatorname{star}(\mathbf{v})$ be the set of all $d$-dimensional polytopes $\Delta \in \mathcal{C}$ containing $\mathbf{v}$ and for such $\Delta$, let $\mathbf{m}_{\Delta}$ be the gradient of $f$ on $\Delta$ (which is just the peg in $[\mathbf{1 3}]$ ). Then the dual polytope of $\mathbf{v}$ with respect to $f$ is defined to be the convex hull of points $\mathbf{m}_{\Delta}$ for all $\Delta \in \operatorname{star}(\mathbf{v})$, which we denote 
by $\widehat{\mathbf{v}}^{f}$ and is denoted by $\Delta(\mathbf{v})^{f}$ in $[\mathbf{1 1}]$. It is a $d$-dimensional (rational) polytope.

Now we are going to prove the following main theorem of this section. Recall that we have the embedding $i_{A}: \mathbb{R}^{d} / \Lambda \hookrightarrow A^{\text {an }}$.

Theorem 3.3. Let $\overline{L_{i}}=\left(L_{i},\|\|_{i}\right)(i=1, \ldots, d)$ be $d$ integrable metrized line bundles on $A$, where \|\|$_{i}$ are toric metrics determined by Green functions $g_{i} \in \mathscr{G}\left(L_{i}\right) \cap \mathscr{C}^{3}\left(\mathbb{R}^{d}\right)$ (cf. Corollary 2.11). Then we have the following equality of measures on $A^{\text {an }}$ :

$$
c_{1}\left(\overline{L_{1}}\right) \wedge \cdots \wedge c_{1}\left(\overline{L_{d}}\right)=\left(i_{A}\right)_{*} \operatorname{Hess}_{g_{1}, \ldots, g_{d}} \mathrm{~d} \mathbf{x} .
$$

Proof. The reader may compare this formula with the one stated in [22, theorem 3.4.2], where the author considers the triple product of curves and measures with singularities.

The proof of the theorem is divided into several steps.

Step 1. First we reduce to the case $\overline{L_{1}}=\cdots=\overline{L_{d}}=\bar{L}=(L,\|\|)$ where $L$ is an ample line bundle on $A$ and \|\| is determined by a Green function $g \in \mathscr{G}_{+}(L) \cap \mathscr{C}^{3}\left(\mathbb{R}^{d}\right)$ such that the matrix $\left(g_{i j}\right)_{i, j=1, \ldots, d}$ is positive definite everywhere. Assuming the theorem holds for this case, consider the subset $\mathscr{G}^{\prime}(A) \subset \mathscr{G}_{\text {int }}(A)$ consisting of such Green functions. Then $g, g^{\prime} \in \mathscr{G}^{\prime}(A)$ implies $a g+b g^{\prime} \in \mathscr{G}^{\prime}(A)$ for $(a, b) \in \mathbb{Z}_{>0}^{2}-\{(0,0)\}$. For any continuous function $f$ on $A^{\text {an }}$, consider the functional

$$
\ell_{f}\left(g_{1}, \ldots, g_{d}\right)=\left(c_{1}\left(\overline{L_{1}}\right) \wedge \cdots \wedge c_{1}\left(\overline{L_{d}}\right)-\left(i_{A}\right)_{*} \operatorname{Hess}_{g_{1}, \ldots, g_{d}} \mathrm{~d} \mathbf{x}\right)(f)
$$

which is symmetric and $\mathbb{Q}$-multilinear in $g_{1}, \ldots, g_{d}$ and $\ell_{f}(g, \ldots, g)=0$ for $g \in \mathscr{G}^{\prime}(A)$ by our assumption. Then for $g_{1}, \ldots, g_{d} \in \mathscr{G}^{\prime}(A)$ and $t_{1}, \ldots, t_{d} \in \mathbb{Z}_{>0}^{d}$,

$$
\begin{aligned}
0 & =\ell_{f}\left(\sum_{i=1}^{d} t_{i} g_{i}, \ldots, \sum_{i=1}^{d} t_{i} g_{i}\right) \\
& =\sum_{\substack{k_{1}, \ldots, k_{d} \geq 0 \\
k_{1}+\cdots+k_{d}=d}} \frac{d !}{k_{1} ! \cdots k_{d} !} \ell_{f}\left(\ldots, g_{i}, \ldots, g_{i}, \ldots\right) t_{1}^{k_{1}} \cdots t_{d}^{k_{d}}
\end{aligned}
$$

where $g_{i}$ appears $d_{i}$ times in the second $\ell_{f}$. Hence $\ell_{f}\left(\ldots, g_{i}, \ldots, g_{i}, \ldots\right)=$ 0 . In particular, $\ell_{f}\left(g_{1}, \ldots, g_{d}\right)=0$ for all $g_{i} \in \mathscr{G}^{\prime}(A)$. But on the other hand, $\mathscr{G}^{\prime}(A)$ generates the whole space $\mathscr{G}_{\text {int }}(A) \cap \mathscr{C}^{3}\left(\mathbb{R}^{d}\right)$ by definition and the existence and smoothness of $g_{\text {can }}$. Then $\ell_{f}\left(g_{1}, \ldots, g_{d}\right)=0$ for all $g_{i} \in \mathscr{G}_{\text {int }}(A) \cap \mathscr{C}^{3}\left(\mathbb{R}^{d}\right)$. The theorem follows.

Step 2. Now fix $g \in \mathscr{G}^{\prime}(A)$ as above and assume $g \in \mathscr{G}_{+}(L)$ for an ample line bundle $L$. By Proposition 2.2, there are $g_{n} \in \mathscr{G}_{\text {for }}(L)$ such that $g_{n} \rightarrow g$ and the corresponding formal $k^{\circ}$-models $\left(\mathcal{X}_{n}, \mathcal{L}_{n}\right)$ satisfying that $\widetilde{\mathcal{L}_{n}}$ is ample on $\widetilde{\mathcal{X}_{n}}$. Hence the models are in fact algebrizable. Now we view $\mathcal{X}_{n}$ as schemes projective and flat over Spec $k^{\circ}$ and $\left(\mathcal{L}_{n}\right)_{\eta} \cong L^{e_{n}}$. We denote the corresponding metrized line bundle determined by $g_{n}$ by 
$\bar{L}_{n}=\left(L,\|\|_{n}\right)$ and by $g$ by $\bar{L}=(L,\|\|)$. By (2.1) and Proposition 1.2 , the measure $c_{1}\left(\bar{L}_{n}\right)^{\wedge d}$ is supported on $i_{A}\left(\mathbb{R}^{d} / \Lambda\right)$ and hence also for their limit. If we let $\mu_{n}$ be the measure $c_{1}\left(\bar{L}_{n}\right)^{\wedge d}$ restricted on $\mathbb{R}^{d} / \Lambda$ and $\mu=\lim _{n} \mu_{n}$, then we only need to prove that $\mu=\mu_{g}$ as elements in $\mathscr{M}\left(\mathbb{R}^{d} / \Lambda\right)$, where $\mu_{g}$ is the measure $\operatorname{Hess}_{g} \mathrm{~d} \mathbf{x} \in \mathscr{M}_{>0}^{3}\left(\mathbb{R}^{d} / \Lambda\right)$.

We claim that for any $\delta>0$, we have

$$
\mu \leq(1+\delta) \mu_{g}
$$

Assuming this, then $\mu \leq \mu_{g}$. But by (2.2) and Lemma 3.2, $|\mu|=\left|\mu_{g}\right|$; i.e., $\left|\mu_{g}-\mu\right|=0$. Hence $\mu=\mu_{g}$, which confirms the theorem.

Step 3. The last step is dedicated to proving the above claim (3.2). We prove that for any $\delta>0$ and $f \in \mathscr{C}_{>0}\left(\mathbb{R}^{d} / \Lambda\right), \mu(f) \leq(1+\delta) \mu_{g}(f)$. Given $\epsilon_{1}>0$, take $\bar{L}_{n}$ as above such that $\left|g_{n}-g\right|<\epsilon_{1}$; then the model $\mathcal{X}_{n}$ determines a rational polytopal decomposition $\left(\mathcal{C}_{n}\right)_{\Lambda}$ of $\mathbb{R}^{d} / \Lambda$ which comes from a $\Lambda$-periodic rational polytopal decomposition $\mathcal{C}_{n}$ of $\mathbb{R}^{d}$. The function $g_{n}$ is rational polytopal and strongly polytopal convex with respect to $\mathcal{C}_{n}$. By Proposition 1.2, the set of irreducible components of $\widetilde{\mathcal{X}_{n}}$ is identified with the set of $\Lambda$-translation classes of vertices in $\mathcal{C}_{n}$. Hence if we denote $Y_{\mathbf{v}}$ the irreducible component corresponding to $\mathbf{v}$, then $Y_{\mathbf{v}}=Y_{\mathbf{v}^{\prime}}$ if and only if $\mathbf{v}=\mathbf{v}^{\prime}+\boldsymbol{\lambda}$ for some $\boldsymbol{\lambda} \in \Lambda$. By (2.1), we have

$$
\mu_{n}=\frac{1}{e_{n}^{d}} \sum_{\mathbf{v} \in \mathfrak{F}} \operatorname{deg} \frac{\mathcal{L}_{n}}{\left(Y_{\mathbf{v}}\right)} \delta_{p(\mathbf{v})}
$$

where we recall that $\mathfrak{F} \subset \mathbb{R}^{d}$ is the fixed fundamental domain of $\Lambda$ and $p: \mathbb{R}^{d} \rightarrow \mathbb{R}^{d} / \Lambda$ is the projection. Here, the multiplicities $m_{j}$ appearing in (2.1) are all 1 since the special fibre is reduced by Proposition 1.2.

We recall a formula in $[\mathbf{1 1}$, p. $366(36)]$ which is deduced from $[\mathbf{9}$, p.112 corollary], that

$$
\operatorname{deg} \overline{\mathcal{L}_{n}}\left(Y_{\mathbf{v}}\right)=d ! \cdot \operatorname{vol}\left(\widehat{\mathbf{v}}^{e_{n} g_{n}}\right)
$$

where $\widehat{\mathbf{v}}^{e_{n} g_{n}}$ is the dual polytope defined before the theorem. Hence we have

$$
(3.3)=d ! \sum_{\mathbf{v} \in \mathfrak{F}} \operatorname{vol}\left(\widehat{\mathbf{v}}^{g_{n}}\right) \delta_{p(\mathbf{v})} .
$$

For any integer $N>0$, we divide $\mathfrak{F}$ into $N^{d}$ blocks as follows. For $\left(b_{1}, \ldots, b_{d}\right) \in\{0,1, \ldots, N-1\}^{d}$, let

$$
\mathfrak{F}_{b_{1}, \ldots, b_{d}}^{(N)}=\left\{\mathrm{x}=x_{1} \boldsymbol{\lambda}_{1}+\cdots+x_{d} \boldsymbol{\lambda}_{d} \mid \frac{b_{i}}{N} \leq x_{i}<\frac{b_{i}+1}{N}\right\} .
$$


Then $\mathfrak{F}=\bigsqcup \mathfrak{F}_{b_{1}, \ldots, b_{d}}^{(N)}$ and $\overline{\mathfrak{F}_{b_{1}, \ldots, b_{d}}^{(N)}}$ is a $d$-dimensional rational polytope. For any $\epsilon_{2}>0$, there exists $N\left(\epsilon_{2}\right)>0$ such that for any $N \geq N\left(\epsilon_{2}\right)$,

$$
\underset{\mathbf{x} \in \mathfrak{F}_{b_{1}, \ldots, b_{d}}^{(N)}}{\max } g_{i j}(\mathbf{x})-\underset{\mathbf{x} \in \mathfrak{F}_{b_{1}, \ldots, b_{d}}^{(N)}}{\min } g_{i j}(\mathbf{x})<\epsilon_{2}
$$

for all $i, j=1, \ldots, d$ and $\left(b_{1}, \ldots, b_{d}\right)$ by uniform continuity. We now assume $N \geq N\left(\epsilon_{2}\right)$ and consider a block, say without lost of generality, $\mathfrak{F}^{(N)}=\mathfrak{F}_{0, \ldots, 0}^{(N)}$. Then

$$
\left.\mu_{n}\right|_{\mathfrak{F}^{(N)}}(f)=d ! \sum_{\mathbf{v} \in \mathfrak{F}^{(N)}} \operatorname{vol}\left(\widehat{\mathbf{v}}^{g_{n}}\right) f(\mathbf{v}) \leq d ! \cdot \operatorname{vol}\left(\Delta^{(N)}\right) \cdot \sup _{\mathbf{x} \in \mathfrak{F}^{(N)}} f(\mathbf{x})
$$

where $\Delta^{(N)}$ is the convex hull of $\mathbf{m}_{\Delta}$ (pegs induced by $g_{n}$ ) for (finitely many) $d$-dimensional polytopes $\Delta \in \mathcal{C}_{n}$ such that $\Delta \cap \mathfrak{F}^{(N)} \neq \emptyset$. Now we are going to give an upper bound for this volume. Let $\mathbf{x}_{0}$ be the point $\frac{1}{2 N}\left(\boldsymbol{\lambda}_{1}+\cdots+\boldsymbol{\lambda}_{d}\right)$, which is the center of symmetry of $\overline{\mathfrak{F}^{(N)}}$. The volume $\operatorname{vol}\left(\Delta^{(N)}\right)$ will keep unchanged under the following operations:

(a): We replace $g_{n}$ by $\tilde{g}_{n}$ where

$$
\tilde{g}_{n}(\mathbf{x})=g_{n}(\mathbf{x})-\nabla g\left(\mathbf{x}_{0}\right) \cdot\left(\mathbf{x}-\mathbf{x}_{0}\right)-g\left(\mathbf{x}_{0}\right) .
$$

(We also let $\tilde{g}(\mathbf{x})=g(\mathbf{x})-\nabla g\left(\mathbf{x}_{0}\right) \cdot\left(\mathbf{x}-\mathbf{x}_{0}\right)-g\left(\mathbf{x}_{0}\right)$.)

(b): We make a translation $\mathrm{x}^{\prime}=\mathbf{x}-\mathrm{x}_{0}$.

(c): We apply a rotation $\mathbf{x}^{\prime \prime}=R \cdot \mathbf{x}^{\prime}$ for some $R \in \mathbf{S O}_{d}$.

Hence we may assume that

(a'): $\nabla g(\mathbf{0})=\mathbf{0} ; g(\mathbf{0})=0$ and $\left|g_{n}-g\right|<\epsilon_{1}$ by (a).

$\left(b^{\prime}\right)$ :

$$
\left(g_{i j}(\mathbf{0})\right)=\left(\begin{array}{llll}
h_{11} & & & \\
& h_{22} & & \\
& & \ddots & \\
& & & h_{d d}
\end{array}\right)
$$

$\left(c^{\prime}\right)$ :

with $h_{g}<h_{11} \leq \cdots \leq h_{d d}<H_{g}$.

$$
\mathfrak{F}^{(N)}=\left\{\mathrm{x}=x_{1} \lambda_{1}^{\prime}+\cdots+x_{d} \boldsymbol{\lambda}_{d}^{\prime} \mid-\frac{1}{2 N} \leq x_{i}<\frac{1}{2 N}\right\}
$$

where $\boldsymbol{\lambda}_{i}^{\prime}=R \cdot \boldsymbol{\lambda}_{i}$ for certain $R \in \mathbf{S O}_{d}$.

For any $\epsilon_{3} \geq 0$, we also introduce the set

$$
\mathfrak{F}_{g, \epsilon_{3}}^{(N)}=\left\{\mathbf{x}^{\prime}=\left(1+\epsilon_{3}\right) \sum_{i=1}^{d} h_{i i} x_{i} \mathbf{e}_{i} \mid \mathbf{x}=\sum_{i=1}^{d} x_{i} \mathbf{e}_{i} \in \mathfrak{F}^{(N)}\right\} .
$$

The following lemma is obvious. 
Lemma 3.4. For any $\mathbf{x}^{\prime} \in \mathfrak{F}_{g, 0}^{(N)}$, the ball $B\left(\mathbf{x}^{\prime}, \frac{\epsilon_{3} h_{g} r_{\mathfrak{F}}}{N}\right)$ is contained in $\mathfrak{F}_{g, \epsilon_{3}}^{(N)}$.

Now for any $\mathbf{x} \in \mathfrak{F}^{(N)}$, we have

$$
g_{i}(\mathbf{x})=\int_{0}^{1} \nabla_{\mathbf{x}} \nabla_{\mathbf{e}_{i}} g(t \mathbf{x}) \mathrm{d} t .
$$

By (3.5), we have

$$
\begin{aligned}
\left|g_{i}(\mathbf{x})-h_{i i} x_{i}\right| & =\left|\int_{0}^{1} \nabla_{\mathbf{x}} \nabla_{\mathbf{e}_{i}} g(t \mathbf{x}) \mathrm{d} t-h_{i i} x_{i}\right| \\
& =\left|\sum_{j=1}^{d} x_{j} \int_{0}^{1} g_{i j}(t \mathbf{x}) \mathrm{d} t-\sum_{j=1}^{d} x_{j} g_{i j}(\mathbf{0})\right| \\
& =\left|\sum_{j=1}^{d} x_{j} \int_{0}^{1}\left(g_{i j}(t \mathbf{x})-g_{i j}(\mathbf{0})\right) \mathrm{d} t\right| \\
& \leq \epsilon_{2}\left(\left|x_{1}\right|+\cdots+\left|x_{d}\right|\right) \leq \frac{\epsilon_{2} \cdot d R_{\mathfrak{F}}}{2 N}
\end{aligned}
$$

The point $\mathbf{x}^{\prime}=h_{11} x_{1} \mathbf{e}_{1}+\cdots+h_{d d} x_{d} \mathbf{e}_{d}$ is in $\mathfrak{F}_{g, 0}^{(N)}$. Let $\mathbf{m}_{\Delta}(\mathbf{x})$ be (any) peg of $\Delta$ containing $\mathbf{x}$; then we have, by Lemma 2.9 ,

$$
\begin{aligned}
\left\|\mathbf{m}_{\Delta}(\mathbf{x})-\mathbf{x}^{\prime}\right\| & \leq\left\|\mathbf{m}_{\Delta}(\mathbf{x})-\nabla g(\mathbf{x})\right\|+\left\|\nabla g(\mathbf{x})-\mathbf{x}^{\prime}\right\| \\
& \leq 2 \sqrt{\epsilon_{1} \cdot H_{g}}+\frac{\epsilon_{2} \cdot d^{\frac{3}{2}} R_{\mathfrak{F}}}{2 N} .
\end{aligned}
$$

Hence, by Lemma 3.4, if we can make choices of $\epsilon_{1}, \epsilon_{2}$, and $\epsilon_{3}$ such that

$$
2 \epsilon_{3} h_{g} r_{\mathfrak{F}} \geq \epsilon_{2} \cdot d^{\frac{3}{2}} R_{\mathfrak{F}}+4 N \sqrt{\epsilon_{1} \cdot H_{g}},
$$

then $\mathbf{m}_{\Delta}(\mathbf{x}) \in \mathfrak{F}_{g, \epsilon_{3}}^{(N)}$. Since the latter is convex, we have vol $\left(\Delta^{(N)}\right) \leq$ $\operatorname{vol}\left(\mathfrak{F}_{g, \epsilon_{3}}^{(N)}\right)=\left(1+\epsilon_{3}\right)^{d} h_{11} \cdots h_{d d} \cdot \operatorname{vol}\left(\mathfrak{F}^{(N)}\right)$. Now we let $\epsilon_{3}=\sqrt[d]{1+\delta}-1$, $\epsilon_{2}=\epsilon_{3} h_{g} r_{\mathfrak{F}} d^{-\frac{3}{2}} R_{\Lambda}^{-1}$. Then for a fixed $N \geq N\left(\epsilon_{2}\right)$, when $n$ is large enough and hence we can take

$$
\epsilon_{1} \leq \frac{1}{H_{g}}\left(\frac{\epsilon_{3} h_{g} r_{\mathfrak{F}}}{4 N}\right)^{2}
$$

we have

$$
\begin{aligned}
\left.\mu_{n}\right|_{\mathfrak{F}^{(N)}}(f) & \leq(1+\delta) d ! \cdot h_{11} \cdots h_{d d} \cdot \sup _{\mathbf{x} \in \mathfrak{F}^{(N)}} f(\mathbf{x}) \\
& \leq(1+\delta) \sup _{\mathbf{x} \in \mathfrak{F}^{(N)}} f \cdot \operatorname{Hess}_{g}(\mathbf{x}) \cdot \operatorname{vol}\left(\mathfrak{F}^{(N)}\right)
\end{aligned}
$$


Summing over all $\left(b_{1}, \ldots, b_{d}\right)$, we have

$$
\mu_{n}(f) \leq(1+\delta) \sum_{\left(b_{1}, \ldots, b_{d}\right)} \sup _{\mathbf{x} \in \mathfrak{F}_{b_{1}, \ldots, b_{d}}^{(N)}} f \cdot \operatorname{Hess}_{g}(\mathbf{x}) \cdot \operatorname{vol}\left(\mathfrak{F}_{b_{1}, \ldots, b_{d}}^{(N)}\right) .
$$

Let $n \rightarrow \infty$ and then $N \rightarrow \infty$; we have

$$
\mu(f) \leq(1+\delta) \int_{\mathfrak{F}} f(\mathbf{x}) \operatorname{Hess}_{g}(\mathbf{x}) \mathrm{d} \mathbf{x},
$$

which confirms the claim (3.2).

q.e.d.

\section{A Calabi-Yau Theorem}

In this section, we state and prove the non-archimedean analogue of the Calabi-Yau theorem for totally degenerate abelian varieties.

4.1. Review of the classical Calabi-Yau theorem. Let us have a quick review of the Calabi conjecture which is proved by Yau in complex geometry. For details, we refer to Yau's original paper [17] and also the book [12, chapter 5] by Joyce. For simplicity, we just state it for the algebraic case. Hence let $M$ be a connected compact complex manifold of dimension $d \geq 1$ and $L$ an ample line bundle on it. Given any smooth metric \|\| on $L$, we have the Chern class $\omega=c_{1}(L,\|\|)$ which is a (smooth) $(1,1)$-form on $M$. It determines a measure, i.e., a top form $\mu=\omega^{\wedge d}$ on $M$. We say \|\| is positive if $\omega$ is positive definite everywhere. Then the measure $\mu$ is obviously positive. The Calabi conjecture asserts that given any smooth positive $(d, d)$-form $\mu^{\prime}$ such that $\int_{M} \mu^{\prime}=\int_{M} \mu$, there exists a smooth positive measure \|\|$^{\prime}$ on $L$, unique up to a scalar, such that $\mu^{\prime}=\left(\omega^{\prime}\right)^{\wedge d}$ where $\omega^{\prime}=c_{1}\left(L,\|\|^{\prime}\right)$.

If we write $\mu^{\prime}=\mathrm{e}^{f} \mu$ for a unique smooth real function $f$ on $M$, then the Calabi conjecture asserts that there exists a unique smooth real function $\phi$ such that

(1): $\omega+\mathrm{dd}^{c} \phi$ is a positive $(1,1)$-form;

(2): $\int_{M} \phi \mu=0$;

(3): $\left(\omega+\mathrm{dd}^{c} \phi\right)^{\wedge d}=\mathrm{e}^{f} \mu$.

If we choose a local coordinate $z_{1}, \ldots, z_{d}$ on an open set $U$ in $M$, then $\left(g_{\alpha \bar{\beta}}\right)_{\alpha, \bar{\beta}=1, \ldots, d}$ is a $d \times d$ hermitian matrix, where $g$ is the Riemannian metric associated with $\omega$. Then the condition (3) reads as

$\left(3^{\prime}\right)$ :

$$
\operatorname{det}\left(g_{\alpha \bar{\beta}}+\frac{\partial^{2} \phi}{\partial z_{\alpha} \partial \bar{z}_{\bar{\beta}}}\right)=\mathrm{e}^{f} \operatorname{det}\left(g_{\alpha \bar{\beta}}\right)
$$

which is a complex Monge-Ampère equation.

More generally, $\omega$ could just be a Kähler form. The existence part of the following theorem is due to Yau and the uniqueness part is due to Calabi. 
Theorem 4.1. Let $M, \omega$ be as above; then

Existence: (cf. [17, $\S 4$, theorem 1]) for any $f \in \mathscr{C}^{k}(M)(k \geq 3)$, there exists $\phi \in \mathscr{C}^{k+1, \alpha}(M)$ for any $\alpha \in[0,1)$ satisfying (1)-(3);

Uniqueness: (cf. [4], [17, $\S 5$, theorem 3]) for any $f \in \mathscr{C}^{1}(M)$, there is at most one $\phi \in \mathscr{C}^{3}(M)$ satisfying (1)-(3).

4.2. A non-archimedean analogue. Recall that we have a totally degenerate abelian variety $A$ of dimension $d$ over $k$ and an ample class $L \in \operatorname{Pic}(A) \otimes_{\mathbb{Z}} \mathbb{Q}$. For any integrable metrized line bundle $\bar{L}=(L,\|\|)$, we define the measure $c_{1}(\bar{L})^{\wedge d}$ on the analytic space $A^{\text {an }}$. Also, we have a skeleton $i_{A}: \mathbb{R}^{d} / \Lambda \hookrightarrow A^{\text {an }}$. The following is a Calabi-Yau theorem in the current setting for positive measures supported on this skeleton, which has certain smoothness in the real-analytic sense.

Theorem 4.2 (Non-archimedean Calabi-Yau). Let $A, L, i_{A}$ be as above. For any $\mu \in \mathscr{M}_{>0}^{k}\left(\mathbb{R}^{d} / \Lambda\right)(k \geq 3)$ such that $|\mu|=\operatorname{deg}_{L}(A)$, there is a semipositive metric \|\| on $L$, unique up to a scalar, such that $c_{1}(\bar{L})^{\wedge d}=\left(i_{A}\right)_{*} \mu$ where $\bar{L}=(L,\|\|)$. Moreover, $\bar{L}$ is toric in the sense of Definition 2.3, whose corresponding Green function $g$ is in $\mathscr{G}_{+}(L) \cap \mathscr{C}^{k+1, \alpha}\left(\mathbb{R}^{d}\right)$ for any $\alpha \in[0,1)$.

Proof. The uniqueness part follows from the general theorem on the uniqueness [18, theorem 1.1.1] proved by Yuan and Zhang.

Now we prove the existence. Recall that we have a canonical Green function $g_{\text {can }}$ for $L$, which determines a measure $\mu_{\text {can }}$ on $\mathbb{R}^{d} / \Lambda$ (which is just $H_{q}$ times the Lebesgue measure). By Theorems 2.4 and 3.3, we only need to prove that for a given $f \in \mathscr{C}^{k}\left(\mathbb{R}^{d} / \Lambda\right)(k \geq 3)$ such that $\int_{\mathbb{R}^{d} / \Lambda} \mathrm{e}^{f} \mathrm{~d} \mathbf{x}=\operatorname{covol}(\Lambda)$, there exists a function $\phi \in \mathscr{C}^{k+1, \alpha}\left(\mathbb{R}^{d} / \Lambda\right)$ for any $\alpha \in[0,1)$ such that:

- The matrix $\left(\left(g_{\mathrm{can}}\right)_{i j}+\phi_{i j}\right)_{i, j=1, \ldots, d}$ is positive definite.

- It satisfies the real Monge-Ampère equation

$$
\operatorname{det}\left(\left(g_{\text {can }}\right)_{i j}+\frac{\partial^{2} \phi}{\partial x_{i} \partial x_{j}}\right)=\frac{H_{q}}{d !} \mathrm{e}^{f} .
$$

- If $f \in \mathscr{C}^{\infty}\left(\mathbb{R}^{d} / \Lambda\right)$, then $\phi \in \mathscr{C}^{\infty}\left(\mathbb{R}^{d} / \Lambda\right)$.

Then we set $g=g_{\text {can }}+\phi$.

We would like to deduce the above PDE problem from the complex case, i.e., Theorem 4.1, as we have promised. Inspired by the formula defining the mixed Hessian, we introduce the following manifold:

$$
\mathbb{A}=\mathbb{R}^{d} / \Lambda \oplus \mathbb{R}^{d} / \Lambda
$$

where we write $\left(x_{1}, \ldots, x_{d} ; y_{1}, \ldots, y_{d}\right)$ for the usual chart. The tangent bundle has a canonical splitting $\mathcal{T}_{\mathbb{A}}=\mathcal{T}_{1} \oplus \mathcal{T}_{2}$ where $\mathcal{T}_{i}$ is the pull-back of the tangent bundle on the $i$-th $\mathbb{R}^{d} / \Lambda$. Write $u_{i}=\partial x_{i}$ and $v_{i}=\partial y_{i}$ and define a complex structure $J$ on $\mathcal{T}_{\mathbb{A}}$ by $J u_{i}=v_{i}, J v_{i}=-u_{i}(i=$ $1, \ldots, d)$. Then as a complex manifold, $\mathbb{A}$ is isomorphic to $\mathbb{C}^{d} /(\Lambda \oplus i \Lambda)$. 
We define

$$
\text { ठ }\left(\left(u_{i}, v_{j}\right),\left(u_{i^{\prime}}, v_{j^{\prime}}\right)\right)=\frac{1}{2}\left(\left(g_{\mathrm{can}}\right)_{i i^{\prime}}+\left(g_{\mathrm{can}}\right)_{j j^{\prime}}\right) .
$$

Then $\partial$ is a Riemannian metric on $\mathbb{A}$ and $\omega\left(w, w^{\prime}\right)=\partial\left(J w, w^{\prime}\right)$ is a Kähler metric on $(\mathbb{A}, J)$ with $\boldsymbol{\mu}=\omega^{\wedge d}$. Define $\mho(\mathbf{x}, \mathbf{y})=f(\mathbf{x})$ which is in $\mathscr{C}^{k}(\mathbb{A})$. Then $((\mathbb{A}, J), \omega, \mho)$ is in the situation of Theorem 4.1. Applying this theorem, we see that there is a unique function $\phi \in \mathscr{C}^{k+1, \alpha}(\mathbb{A})$ for any $\alpha \in[0,1)$ satisfying (1)-(3). If we write the Monge-Ampère equation (4.1) explicitly in the current situation, we see that $\boldsymbol{\phi}$ satisfies

$$
\operatorname{det}\left(\partial_{\alpha \bar{\beta}}+\frac{\partial^{2} \boldsymbol{\phi}(\mathbf{x}, \mathbf{y})}{\partial \mathbf{z}_{\alpha} \partial \overline{\mathbf{z}}_{\bar{\beta}}}\right)=\frac{H_{q}}{d !} \mathrm{e}^{f(\mathbf{x})}
$$

where $\mathbf{z}_{\alpha}=\mathbf{x}_{\alpha}+i \mathbf{y}_{\alpha}$ and $\overline{\mathbf{z}}_{\bar{\beta}}=\mathbf{x}_{\bar{\beta}}-i \mathbf{y}_{\bar{\beta}}$. For any $\mathbf{y}_{0} \in \mathbb{R}^{d} / \Lambda$, let $\phi_{\mathbf{y}_{0}}(\mathbf{x}, \mathbf{y})=\boldsymbol{\phi}\left(\mathbf{x}, \mathbf{y}-\mathbf{y}_{0}\right)$. Then $\boldsymbol{\phi}_{\mathbf{y}_{0}}$ is also a $\mathscr{C}^{k+1, \alpha}$-solution satisfying (1)-(3). Hence by the uniqueness, $\boldsymbol{\phi}_{\mathbf{y}_{0}}=\boldsymbol{\phi}$ for any $\mathbf{y}_{0}$, i.e.,

$$
\frac{\partial \phi}{\partial \mathbf{y}_{i}} \equiv 0 ; \quad i=1, \ldots, d
$$

Restricting to $\mathbb{R}^{d} / \Lambda \times\{\mathbf{0}\}$, we see that $\phi(\mathbf{x}):=\boldsymbol{\phi}(\mathbf{x}, \mathbf{0}) \in \mathscr{C}^{k+1, \alpha}\left(\mathbb{R}^{d} / \Lambda\right)$ for any $\alpha \in[0,1)$, satisfies the real Monge-Ampère equation (4.2) and such that $\left(\left(g_{\text {can }}\right)_{i j}+\phi_{i j}\right)$ is positive definite. Hence the theorem is proved.

q.e.d.

\section{References}

[1] V.G. Berkovich, Spectral theory and analytic geometry over non-archimedean fields, Mathematical Surveys and Monographs, 33. American Mathematical Society, Providence, RI, 1990. MR 1070709, Zbl 0715.14013.

[2] V.G. Berkovich, Vanishing cycles for formal schemes, Invent. Math. 115 (1994) 539-571. MR 1262943, Zbl 0791.14008.

[3] S. Bosch \& W. Lütkebohmert, Degenerating abelian varieties, Topology 30 (1991) 653-698. MR 1133878, Zbl 0761.14015.

[4] E. Calabi, The space of Kähler metrics, Proceedings of the International Congress of Mathematicians, Amsterdam, 1954. Vol. 2. 206-207, North-Holland, Amsterdam, 1956. MR 70535, Zbl 0077.00303.

[5] A. Chambert-Loir, Mesure et équidistribution sur les espaces de Berkovich, J. Reine Angew. Math. 595 (2006) 215-235. MR 2244803, Zbl 1112.14022.

[6] A. Chambert-Loir \& A. Thuillier, Mesures de Mahler et équidistribution logarithmique. Annales de l'Institut Fourier 59 (2009) 977-1014. MR 2543659, Zbl 1192.14020 .

[7] S.-Y. Cheng \& S.-T. Yau, The real Monge-Ampère equation and affine flat structures, In Proceedings of the 1980 Beijing Symposium on Differential Geometry and Differential Equations, Vol. 1. 339-370, Science Press, Beijing, 1982. MR 0714332 . 
[8] J. Fresnel \& M. van der Put, Rigid analytic geometry and its applications, Progress in Mathematics, 218. Birkhäuser Boston, Inc., Boston, MA, 2004. MR 2014891, Zbl 1096.14014.

[9] W. Fulton, Introduction to toric varieties, Annals of Mathematics Studies, 131. The William H. Roever Lectures in Geometry. Princeton University Press, Princeton, NJ, 1993. MR 1234037, Zbl 0813.14039.

[10] W. Gubler, Local heights of subvarieties over non-Archimedean fields, J. Reine Angew. Math. 498 (1998) 61-113. MR 1629925, Zbl 0906.14013.

[11] W. Gubler, Tropical varieties for non-Archimedean analytic spaces, Invent. Math. 169 (2007) 321-376. MR 2318559, Zbl 1153.14036.

[12] D.D. Joyce, Compact manifolds with special holonomy, Oxford Mathematical Monographs. Oxford University Press, Oxford, 2000. MR 1787733, Zbl 1027.53052 .

[13] P. McMullen, Duality, sections and projections of certain Euclidean tilings, Geom. Dedicata 49 (1994) 183-202. MR 1266273, Zbl 0796.52004.

[14] D. Mumford, Abelian varieties, Tata Institute of Fundamental Research Studies in Mathematics, 5. Oxford University Press, Oxford, 1970. MR 2514037, Zbl 1177.14001 .

[15] D. Mumford, An analytic construction of degenerating abelian varieties over complete rings, Compos. Math. 24 (1972) 239-272. MR 0352106, Zbl 0241.14020.

[16] S.-T. Yau, On Calabi's conjecture and some new results in algebraic geometry, Proceedings of the National Academy of Sciences of the U.S.A. 74 (1977) 17981799.

[17] S.-T. Yau, On the Ricci curvature of a compact Kähler manifold and the complex Monge-Ampère equation, I, Commun. Pure Appl. Math. 31 (1978) 339-411. MR 0480350, Zbl 0369.53059.

[18] X. Yuan \& S. Zhang, Calabi-Yau theorem and algebraic dynamics, preprint, at http://www.math.columbia.edu/ szhang/papers/Preprints.htm.

[19] S. Zhang, Admissible pairing on a curve, Invent. Math. 112 (1993) 171-193. MR 1207481, Zbl 0795.14015.

[20] S. Zhang, Positive line bundles on arithmetic varieties, J. Am. Math. Soc. 8 (1995) 187-221. MR 1254133, Zbl 0861.14018.

[21] S. Zhang, Small points and adelic metrics, J. Alg. Geom. 4 (1995) 281-300. MR 1311351, Zbl 0861.14019.

[22] S. Zhang, Gross-Schoen cycles and dualizing sheaves, Invent. Math. 179 (2010) 1-73. MR 2563759, Zbl 1193.14031.

Department of Mathematics

COLUMBia University

NEW YORK NY 10027

E-mail address: liuyf@math.columbia.edu 